\title{
„Vor allem - da erste Gedanke is ja oft auch gar nicht so gmeint“6 Operatoren zur Charakterisierung des kommunikativen und mentalen Status von Äußerungen im sozialen Raum
}

\author{
Ann Kathrin Fischer und Kristina Herbert (Graz)
}

\begin{abstract}
This paper investigates discursive structures of spoken language in formal and informal communication settings (almost 62 hours of recorded time) of urban speakers in Austria's two biggest cities Vienna and Graz and their surroundings. We focus on so-called bipartite "OperatorSkopus-Strukturen" ('operator-scope-structures'), examining their pragmatic and discourse organising function within specific conversational situations. Our main aim is to offer in-depth analyses of these structures by characterising the mental and communicative status of such utterances. Starting out with a discussion of characteristics of spoken language research, especially by arguing for the replacement of the grammatical concept of sentences with the interactional concept of turn-constructional units, we address the phenomenon of discourse markers and Operator-Skopus-Strukturen in particular. This is followed by the description of our data set and the subsequent analyses and discussion of the selected examples. According to our findings, Operator-Skopus-Strukturen appear in both conversational settings and among all groups of speakers. We demonstrate that all speakers use the structures with the same pragmatic function of giving information to their conversation partner on how to interpret and understand the message behind the utterance. Within this scope, the speaker's intention can vary depending on the conversation partner, the topic of the conversation or the situation.
\end{abstract}

\section{$1 \quad$ Einleitung}

Im vorliegenden Beitrag stehen syntaktische Strukturen gesprochener Sprache im Vordergrund, die spezielle pragmatische Funktionen im Diskurs einnehmen. Dazu gehört beispielsweise das Verdeutlichen von formalen oder inhaltlichen Relationen und Zusammenhängen von Äußerungen im Diskurs oder auch die konkrete Nennung des Handlungstyps, welcher mit der Äußerung vollzogen werden soll. Die sprachlichen Einheiten, die dafür Verwendung finden, wurden in der Forschung bereits auf unterschiedliche Art und Weise beschrieben, terminologisch gefasst und abgehandelt. So gibt es beispielsweise im anglo-amerikanischen Raum die Bezeichnung discourse marker (cf. Fraser 1990; Schiffrin 1987; Lenk 1998), von der sich die Kategorie der Diskursmarker für das Deutsche ableitet. Zusammengefasst werden solche diskurs-strukturierenden Einheiten auch unter „Konstruktionen am Satzrand“, zu der „Herausstellung“ (cf. Altmann 1981) und „Vorvorfeldbesetzung“ (cf. Auer 1997) zu rechnen sind. Eine andere, weiter 
gefasste Klassifikation nehmen Barden/Elstermann/Fiehler (cf. 2001) vor, wenn sie von sogenannten Operator-Skopus-Strukturen sprechen, in denen der Operator eine Verstehensanleitung oder -anweisung für den nachfolgenden Skopus liefert (cf. Barden/Elstermann/Fiehler 2001: 197). Im Folgenden sollen diese unterschiedlichen Konzepte und Klassifizierungen näher beleuchtet werden, bevor ein Ansatz für die Analyse der eigenen Daten ausgewählt und zur Anwendung gebracht wird.

Korpuslinguistische Forschung, deren Datenbasis aus gesprochener Sprache besteht, kann sich schwerlich einer Diskussion des Satzbegriffs erwehren. Diese stellt die Grundlage für die weiterführende Beschreibung von syntaktischen Besonderheiten im Diskurs dar. Ausgehend davon, dass gesprochener Sprache nicht einfach die normgrammatischen Regeln der geschriebenen Sprache zugrunde gelegt werden können, ist es unabdingbar, den Rahmen entsprechend abzustecken. Aus diesem Grund wird im ersten Abschnitt des Beitrags (Kapitel 2) auf Spezifika der gesprochenen Sprache Bezug genommen und anschließend der Satz- bzw. Einheitenbegriff diskutiert. Im Weiteren steht die Forschung zu Diskursmarkern (Kapitel 2.1) im Mittelpunkt des Interesses, gefolgt von der Einordnung der Operator-Skopus-Strukturen und der Konzentration auf Operatoren zur Charakterisierung des kommunikativen und mentalen Status (Kapitel 2.2). Darauf folgen eine Beschreibung des Korpus, der Datenerhebung und -aufbereitung (Kapitel 3) sowie die Analyse und Präsentation der Ergebnisse. Die Ergebnisdiskussion vor dem Hintergrund theoretischer Konzepte (Kapitel 4) bildet, gefolgt von einem kurzen Fazit (Kapitel 5), den Abschluss des Beitrags.

\section{Gesprochene-Sprache-Forschung und Grammatiktheorie}

Die Gesprochene-Sprache-Forschung ist in der deutschsprachigen sprachwissenschaftlichen Forschungslandschaft erst seit ca. 55 Jahren Thema (erste Arbeiten von Rupp (1965) und Leska (1965)) und obwohl es mittlerweile zahlreiche Analysen mündlicher Sprachproduktion gibt, existiert keine einheitliche Grammatik der gesprochenen Sprache. Allerdings gibt es unterschiedliche Ansätze und Zugänge zur Beschreibung einer solchen. Darunter fällt beispielsweise die Construction Grammar, die nach Deppermann auf der Inadäquatheit folgender drei Prämissen strukturalistischer Grammatiktheorien fußt:

Satz-Prämisse: Vollständige syntaktische Einheiten sind Sätze; sie drücken eine Proposition aus und bestehen mindestens aus Subjekt und Prädikat.

Formalitätsprämisse: Syntaktische Regeln sind rein formal, und deshalb abstrakt und allgemein.

Sie sind deduktiv und exhaustiv, i. e., sie gelten für alle Instanzen der betreffenden grammatischen Kategorie (Wortart, Satztyp etc.) bzw. syntaktischen Relation (Satzglied etc.).

Kompositionalitätsprämisse: Phrasen- und Satzbedeutung sind kompositional: Sie ergeben sich aus der lexikalischen Bedeutung der Wörter und der syntaktischen Struktur der Verknüpfung.

(Deppermann 2006: 44, Hervorhebungen i. O.)

Da gesprochene Sprache durchsetzt ist von Äußerungskonstruktionen wie Anakoluth, Aposiopese und Ellipse oder sich auch diverse Gliederungsmerkmale beobachten lassen, kann den zuvor genannten Prämissen somit in dieser Form nicht entsprochen werden. Diese Konstruktionen machen außerdem deutlich, dass der Satzbegriff an sich bereits problematisch ist beziehungsweise nicht gleichermaßen wie für die geschriebene Sprache auch auf spontansprachlichen, mündlichen Sprachgebrauch angewendet werden kann. 
Hennig (cf. 2006: 177) hält beispielsweise als ausschlaggebendes Kriterium das Vorhandensein eines finiten Verbs fest, um Sätze von anderen syntaktischen Strukturen abzugrenzen. Sie stellt einen Definitionsversuch an, indem sie formuliert: „Eine syntaktische Einheit ist dann ein Satz, wenn a) ein finites Verb bzw. eine komplexe verbale Struktur vorhanden ist und die Valenzpotenz des Valenzträgers regulär realisiert wird und b) diese Realisierung in kontinuierlicher Weise erfolgt, i. e. wenn keine Planungsschwierigkeiten an der Satzoberfläche zu erkennen sind." (Hennig 2006: 178)

Sie führt diese Eingrenzung in folgender Beschreibung noch näher aus:

Ein kanonischer Satz, i. e. ein Satz, der sowohl in geschriebener als auch in gesprochener Sprache vorkommen kann und der Indifferenzgrammatik des Systems angehört, muss die Kriterien a) und b) erfüllen. Wenn das Kriterium a) erfüllt ist, aber das Kriterium b) nicht, handelt es sich um einen auf Grund der Parameter des Nähesprechens möglichen Satz, der sich durch die Beschreibungskategorie „möglicher Satz“ erfassen lässt. Da der „mögliche Satz“ in gesprochener Sprache ja keineswegs unkanonisch ist und da der kanonische, a) und b) erfüllende Satz ja keineswegs unmöglich ist, schlage ich folgende Differenzierung vor:

Kriterium a) + Kriterium b) = kanonischer Satz prototypischer geschriebener Sprache

Kriterium a) = kanonischer Satz prototypischer gesprochener Sprache

(Hennig 2006: 181, Hervorhebungen i. O.)

Diese Einteilung gemäß dem sprachlichen Medium, in welchem ein Satz realisiert wird, ermöglicht zwar eine differenziertere Verwendung des Satzbegriffs. Sie stellt allerdings noch keine ausreichende Definition dar, die alle Komponenten berücksichtigt. Bei Hennigs Ansatz werden alle Äußerungseinheiten ausgespart, die kein finites Verb oder eine komplexe verbale Struktur aufweisen. Diese gibt es aber in gesprochener Sprache häufig. Trifft also Kriterium a) nach Hennig nicht zu, ist nach ihrer Definition kein Satz vorhanden. Eine Beschränkung auf formale grammatische Kriterien wäre bei der Analyse von Gesprochene-Sprache-Daten jedoch zu kurz gedacht und so müssen ebenso Intonation und Prosodie berücksichtigt werden. Darüber hinaus weist Schlobinski darauf hin: „Bei der Analyse syntaktischer Strukturen der gesprochenen Sprache spielen neben intonatorischen Faktoren insbesondere pragmatische eine Rolle. Art des Diskurstyps, die Ebene der Sprechhandlungen, Konversationsstrukturen und konversationelle Implikaturen sind zentrale, die syntaktische Variation erklärende Bedingungsfaktoren." (Schlobinski 1997: 19) Hiermit wird bereits deutlich, welch komplexe und multivariate Gebilde Strukturen gesprochener Sprache sind und warum eine einheitliche Beschreibung beziehungsweise eine Grammatik der gesprochenen Sprache ein schwieriges Unterfangen darstellt.

Auch Deppermann zielt auf diesen Punkt ab, wenn er anführt:

Gesprächsanalyse und Construction Grammar lehnen es beide ab, Syntax als autonom organi-
siertes Modul zu betrachten und entsprechend zu analysieren. Es ist gerade ein Leitsatz der Inter-
aktionalen Linguistik, den holistischen Zusammenhang von Syntax, Semantik, Prosodie, Prag-
matik, Multimodalität und sequenziell-kontextuellen Aspekten grammatischer Strukturen im Ge-
spräch zu rekonstruieren und dabei den Kontingenzen des empirischen Datums in all seiner
Reichhaltigkeit Rechnung zu tragen (cf. Schegloff/Ochs/Thompson 1996).

(Deppermann 2006: 59, Hervorhebungen i. O.)

Um Syntax in gesprochener Sprache zu analysieren und mehrere Faktoren miteinzubeziehen, können nicht ausschließlich die normgrammatischen syntaktischen Regeln für geschriebene 
Sprache angewendet werden. Vielmehr spricht Schlobinski in diesem Zusammenhang von der Notwendigkeit einer „radikalen Pragmatisierung der Syntaxschreibung“ (1997: 11).

Dazu, was eine pragmatische Syntax ausmacht, schreibt Jürgens (1999: 20):

Eine pragmatische Syntax sollte sich dadurch auszeichnen, daß sie die in Texten und Diskursen regelhaft verwendeten sprachlichen Strukturen zu ihrem Gegenstand erhebt und diese Strukturen mit Blick auf die kommunikativen Gegebenheiten der Äußerung beschreibt. Daraus ergibt sich zwangsläufig die Forderung, den Zusammenhang der verschiedenen inner- und außersprachlichen Faktoren, insbesondere die kognitiven Grundlagen der Textproduktion und -rezeption sowie Fragen des (sprachlichen und außersprachlichen) Kontextes, nicht nur einzubeziehen, sondern zentral zu berücksichtigen.

Jürgens (1999: 20)

Es bedarf also einer Neuorientierung, weg vom klassischen normgrammatischen Satzbegriff. Weitet man den Blick, stößt man auf die Grundannahme der Construction Grammar, die lautet: „Constructions, not categories and relations, are the basic, primitive units of syntactic representation" (Croft 2001: 46). So stellt auch Fiehler (cf. 2005: 1175) in der Unterscheidung der Grundeinheiten der gesprochenen und geschriebenen Sprache dem Satzbegriff der Schriftlichkeit die Begriffe „funktionale Einheit“ und „Gesprächsbeitrag“ der Mündlichkeit gegenüber.

Funktionale Einheiten sind die kleinsten Bestandteile des [Gesprächs-]Beitrags, denen die Gesprächsbeteiligten im Prozess der Produktion und Rezeption der Beiträge eine separate Funktion im und für den Kommunikationsprozess zuschreiben können. Die Untereinheiten des Beitrags werden damit primär funktional, nicht syntaktisch oder prosodisch bestimmt. Die Identifizierung funktionaler Einheiten macht von syntaktischen und prosodischen Mitteln unterstützenden Gebrauch, sie ist aber weder allein noch primär von ihnen abhängig.

(Fiehler 2005: 1232f.)

Couper-Kuhlen/Selting (2001: 3) halten in Bezug auf die Interaktionale Linguistik außerdem fest: „It treats speech as an ongoing and emergent product in a social semiotic event and language as providing one set of resources for the accomplishment of goals and tasks within this event." Sprache nimmt demgemäß dynamischen Charakter in der Interaktion an und stellt eine Komponente dar, in der Interaktion einen bestimmten Zweck durch die Sprechhandlung zu erfüllen.

In Anlehnung daran wollen wir nicht von Sätzen, sondern vielmehr von „Konstruktionen“ oder „funktionalen, interaktiven Einheiten“ im Sinne von ,turn-constructional units“ (cf. Schegloff 2007: 4) als analytische Grundkategorie für unsere Gesprächsdaten sprechen.

\subsection{Phänomenbereich Diskursmarker}

Im Fokus unseres Interesses stehen kurze Wörter oder verfestigte Phrasen am Beginn von Äußerungen, die eine pragmatische Funktion erfüllen. Sie eröffnen eine zusätzliche Dimension im Sinne eines vorausgehenden Hinweises darauf, wie die anschließende Äußerung verstanden werden soll. Solche diskursstrukturierenden sprachlichen Strukturen sind bereits auf unterschiedliche Weise gefasst und mit verschiedenen Bezeichnungen versehen worden. Es handelt sich um ein Phänomen, das im deutschsprachigen Raum beispielsweise in Untersuchungen zu „Diskursmarkern“ (Gohl/Günthner 1999; Günthner/Imo 2003; Imo 2007, 2012) in Erscheinung getreten ist, aber auch unter den Begriffen „Gliederungssignale“ (Gülich 1970; Weinrich 1993; 
Helbig/Buscha 2001), „nichtpropositionale Konnektoren“ (Pasch et al. 2003: 369) oder „VorVorfeldausdrücke“ (Thim-Mabrey 1988) bekannt ist. In der englischsprachigen Forschungslandschaft finden sich dafür Bezeichnungen wie ,pragmatic markers“ (Brinton 1998), „discourse markers“ (Fraser 1990; Shiffrin 1987; Lenk 1998; Fischer 2014), ,,pragmatic expressions“ (Erman 1987) oder „discourse particles“ (Schourup 1982, 1999; Kroon 1995). Was diese Vielzahl an Bezeichnungen und Konzepten vereint, ist die Einordnung nach einem definitorisch dominierenden Merkmal, das entweder aus der diskursorganisierenden bzw. interpersonalen Funktion oder dem formalen Kriterium der äußerungsinitialen Position besteht (cf. Imo 2016: 3). Imo fasst Diskursmarker und ihre Abgrenzung gegenüber anderen Kategorien wie folgt zusammen:

Diskursmarker haben pragmatische und diskursstrukturierende Funktionen, sie sind semantisch verblasst und aus semantischer Sicht daher weglassbar, sie haben eine ,Scharnierfunktion zwischen Äußerungen“ (Fiehler 1999 spricht in diesem Zusammenhang von „Operator-SkopusStrukturen"), und sie besetzen im Deutschen die Vor-Vorfeldposition [...]. Das Kriterium der Vor-Vorfeldbesetzung ist im Deutschen entscheidend für die Abgrenzung der Kategorie „Diskursmarker“ von den „Gesprächspartikeln“, „pragmatischen Markern“, „Gliederungssignalen“ etc., die ähnliche Funktionen wahrnehmen, aber in ihrer Stellung nicht eingeschränkt sind.

(Imo 2007: 6)

Zur Gesprächsorganisation beziehungsweise Herstellung von Diskurskohärenz steht die sequenzielle Funktion der kurzen Wörter oder Phrasen im Vordergrund, es können aber auch andere pragmatische Funktionen wie das Erzeugen von Aufmerksamkeit, die Rederechtseinforderung oder Themenorganisation vorherrschend sein. Dieser Priorisierung folgt auch der Ansatz der Operator-Skopus-Strukturen, wie er von Barden/Elstermann/Fiedler (2001) vertreten wird. Hier steht die pragmatische Funktion des Bereitstellens einer Verstehensanleitung oder Verstehensanweisung für eine Äußerung (Skopus) durch einen Operator im Zentrum ihres theoretischen Ansatzes. Es soll im Folgenden dieser Ansatz der Operator-Skopus-Strukturen genauer vorgestellt werden, da er die Basis für die nachfolgenden Analysen und den Ausgangspunkt für die theoretische Weiterentwicklung des Konzepts darstellt.

\subsection{Operator-Skopus-Strukturen}

Unter Operator-Skopus-Strukturen versteht man ,spezifische zweigliedrige sprachliche Einheiten, deren einer Bestandteil, der Operator, aus einem Wort oder einer kurzen Formel besteht [...], und deren anderer Bestandteil, der Skopus, eine vollständige Äußerung darstellt." (Barden/Elstermann/Fiehler 2001: 197) Diese Definition macht deutlich, dass der Operator allein keine selbständige interaktive Einheit darstellt und somit auch nicht isoliert vorkommen kann. Erst in Kombination mit dem Skopus ergibt sich eine vollständige Äußerung. Der Operator, der - klassischerweise, aber nicht zwingend - dem Skopus vorangestellt ist, hat eine projektive Kraft, indem er eine gewisse Erwartungshaltung aufbaut, wie die nachfolgende Äußerung im Skopus zu verstehen ist. Er fungiert somit als eine Hilfestellung für den/die Rezipienten/Rezipientin, die Äußerung so, wie sie vom Sprecher/von der Sprecherin intendiert ist, aufzufassen und zu interpretieren. „Bei der Operator-Skopus-Struktur handelt es sich also um eine pointiert zweigliedrige Art der hörerorientierten Organisation von Information." (Barden/Elstermann/Fiehler 2001: 198) 
Formal gesehen wird der Operator entweder als Nomen, nominaler Komplex oder Matrixsatz realisiert. Letztgenannte Konstruktion setzt einen asyndetischen Anschluss der Äußerung mit Verbzweitstellung voraus. Matrixsätze mit der subordinierenden Konjunktion dass entsprechen demgemäß keiner Operator-Skopus-Struktur (cf. Barden/Elstermann/Fiehler 2001: 205f.). Die bereits angesprochene Zweigliedrigkeit von Operator-Skopus-Strukturen ergibt sich daraus, dass der Operator syntaktisch abgehoben ist durch Voranstellung (äußerungsinitial), insertierte oder nachgestellte Position. Aber auch durch prosodische Markierung bzw. Hervorhebung kann diese zweigliedrige Struktur erzeugt werden. Dazu können sich SprecherInnen unterschiedlicher prosodischer Auszeichnungen bedienen. Drei Positionen, an denen mittels Prosodie die Struktur abgesetzt werden kann, sind nach Barden/Elstermann/Fiehler (2001: 208) folgende:

- vor dem Operator, um zu signalisieren, dass eine neue Einheit beginnt,

- nach dem Operator, um zu signalisieren, dass es sich um die zweigliedrige OperatorSkopus-Struktur handelt und

- am Ende des Skopus, um das Ende der Operator-Skopus-Struktur zu markieren.

Die prosodische Markierung kann dabei durch unterschiedliche Mittel erfolgen. Dazu gehören etwa Intonationskontur/-einheit, Akzent, Pausen, finaler Tonhöhenverlauf, Tonhöhenansatz, Tonhöhensprünge, Variationen von Lautstärke, Sprechgeschwindigkeit und Tonhöhenregister sowie weitere Phänomene wie z. B. Dehnung von finalen Vokalen, ein Glottisverschluss oder Wortfragmente als Grenzsignale (cf. Fiehler et al. 2004: 312f.). „Die Tonhöhenbewegungen werden am häufigsten als Mittel zur Segmentierung zwischen Operator und Skopus eingesetzt.” (Barden/Elstermann/Fiehler 2001: 216)

Die prosodische Absetzung der Struktur beziehungsweise der Elemente Operator und Skopus ist zumeist eine fakultative zusätzliche Auszeichnung. Es gibt aber auch Formen, die obligatorisch eine prosodische Markierung erfordern, um überhaupt in Operatorfunktion auftreten zu können, da weder Syntax noch Semantik eine Zweigliedrigkeit erkennen und damit auf eine Operator-Skopus-Struktur schließen lassen (cf. Barden/Elstermann/Fiehler 2001: 212). Das zeigt sich vor allem bei koordinierenden Konjunktionen (wie und, denn, oder) sowie bei Partikeln (wie also), die als Gliederungssignale fungieren.

Zusammenfassend lässt sich sagen, dass die prosodische Hervorhebung der Operator-Skopus-
Struktur zwei Funktionen hat: zum einen dient sie dazu, Strukturen zu eindeutigen Operator-
Skopus-Strukturen zu machen (bei prosodisch obligatorisch markierten Operatoren), und zum
anderen bekommt die Struktur durch diese Hervorhebung ein zusätzliches kommunikatives Ge-
wicht (bei prosodisch fakultativ markierten Operatoren).

(Barden/Elstermann/Fiehler 2001: 217)

Die funktionale Komponente von Operator-Skopus-Strukturen ist die der Verstehensanweisung oder -anleitung für den Hörer/die Hörerin. Diese Hauptfunktion, die der Operator einnimmt, wird von Barden/Elstermann/Fiehler (cf. 2001: 204) in vier zentrale Bereiche aufgegliedert:

1. Typ der sprachlichen Handlung: Der Operator kann vorneweg den Handlungstyp der Äußerung verdeutlichen, indem er diesen entweder explizit benennt oder auch nur usuell mit ihm assoziiert wird (cf. Barden/Elstermann/Fiehler 2001: 204);

2. Ausgedrückter mentaler Status: Der Operator verdeutlicht den psychisch-mentalen Status einer Äußerung, indem dieser durch Benennung expliziert wird (ich meine, ich weiß, ich 
glaube etc. als Matrixsatz mit asyndetischem Anschluss und V2-Stellung) (cf. Barden/Elstermann/Fiehler 2001: 205f.);

3. Kommunikativer Status der Äußerung im Skopus: Der Operator charakterisiert die Äußerung im Skopus hinsichtlich ihrer Geltung, Relevanz, Offenheit oder Modalität (cf. Barden/Elstermann/Fiehler 2001: 206f.);

4. Relationen bzw. Zusammenhänge im Diskurs: Der Operator verdeutlicht die Beziehungen zwischen der Bezugsäußerung und anderen Äußerungen im Diskurs. Dies kann sowohl formale Aspekte der Gesprächsorganisation betreffen, aber auch inhaltlich-funktionale Relationen aufzeigen (cf. Barden/Elstermann/Fiehler 2001: 207).

Im Weiteren wird das Augenmerk auf die Operatoren aus den Bereichen 2 und 3 gerichtet, da diese im Fokus des Analyseteils des Artikels stehen. Die Verdeutlichung des mentalen Status der Äußerung im Skopus bezieht sich auf das Ausdrücken von Meinungen, (Ein-)Schätzungen, Glauben oder Wissen. Durch die Verwendung eines entsprechenden Operators zur Charakterisierung des mentalen Status (z. B. ich meine/glaube/weiß/denke/finde etc.) wird der Äußerung eine Verstehensanleitung vorangestellt, die dem Hörer/der Hörerin die Interpretation des Gesagten erleichtert beziehungsweise abnimmt. Der Operator reduziert die Transferleistung des Gesprächspartners/der Gesprächspartnerin, indem er gleich zu Beginn der Äußerung auf deren psychisch-mentale Qualität verweist.

Der kommunikative Status der Äußerung wird dem Gegenüber mittels Operatoren signalisiert, die den Stellenwert der Äußerung im Skopus ausdrücken. Es können auf diese Weise die Geltung, die Relevanz, die Offenheit und die Modalität von Äußerungen verdeutlicht werden. Wird auf die Geltung abgehoben, kann dies durch den Verweis auf den Wahrheitsgehalt einer Äußerung oder die Sicherheit, mit der diese ausgedrückt wird, geschehen. Operatoren wie ehrlich, wirklich, tatsächlich sowie sicher, gewiss, Tatsache ist etc. finden in dieser Funktion Verwendung. Um die Relevanz einer Äußerung im Skopus zu charakterisieren, dienen unter anderem Operatoren wie wichtig(er), vor allem, vor allen Dingen. Offenheit wird mittels ehrlich/offen gesagt, unter uns, im Vertrauen etc. ausgedrückt und Modalität beispielsweise durch im Ernst oder Spaß beiseite.

In Anlehnung an Barden/Elstermann/Fiehler (cf. 2001) legen wir obligatorische Kriterien zur Identifizierung von Operator-Skopus-Strukturen fest. Diese Kriterien sind in der nachfolgenden Überblicksgrafik dargestellt und werden im Weiteren genauer erläutert. 
Operator-Skopus-Struktur

Operator ist

prosodisch oder

syntaktisch

abgehoben

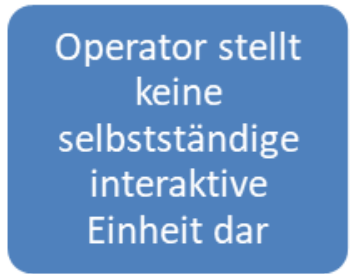

Operator und

Skopus bilden

zweigliedrige,

asyndetische

Struktur

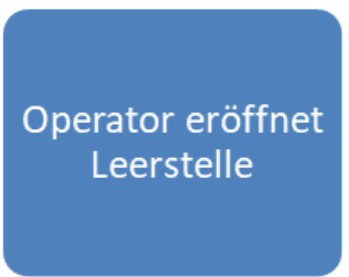

Operator hat

projektive Kraft

auf nachfolgende

Äußerung

\section{Abbildung 1: Definitorische Kriterien zur Identifikation von Operator-Skopus-Strukturen}

Eine Operator-Skopus-Struktur liegt nach unserem Verständnis nur dann vor, wenn

1. der Operator prosodisch oder syntaktisch abgehoben ist. Das bedeutet, dass der Operator z. B. durch Pause, Fokusakzent oder Tonhöhenbewegung vom Skopus abgesetzt oder syntaktisch nicht vollständig in die Äußerung integriert ist. Daraus folgt, dass Operator und Skopus immer eine zweigliedrige, asyndetische Struktur bilden.

2. der Operator allein keine selbstständige interaktive Einheit darstellt. Erst mit dem Skopus zusammen konstituiert er eine interaktive Einheit. Es besteht damit eine hierarchische Struktur zwischen Operator und Skopus. Das bedeutet gleichzeitig, dass der Operator immer eine Leerstelle eröffnet und eine Äußerung, die nur aus einem Operator besteht, noch nicht abgeschlossen ist beziehungsweise nicht allein stehen kann (im Gegensatz zum Skopus).

3. der Operator Projektionskraft auf die nachfolgende Äußerung hat. Die eröffnete Leerstelle muss syntaktisch und semantisch aufgefüllt werden. Aus dem leiten wir auch ab, dass wir solche Fälle ausschließen, die zwar rückwirkend eine projektive Kraft haben können, allerdings die anderen Kriterien nicht erfüllen.

Diese Kriterienaufstellung macht deutlich, dass die Operator-Skopus-Struktur ,durch das Zusammenspiel von funktionalen und formalen Eigenschaften gekennzeichnet ist und dementsprechend auch nur unter gleichzeitiger Berücksichtigung ihrer funktionalen Leistungen und formalen Eigenschaften rekonstruiert werden kann.” (Barden/Elstermann/Fiehler 2001: 200) Es müssen im Rahmen der vorliegenden Studie alle hier angeführten Vorgaben erfüllt werden, um eine Äußerung als Operator-Skopus-Struktur zu klassifizieren.

Mit Blick auf die von uns festgelegten Kriterien, bewerten wir nur diejenigen Konstruktionen als Operator-Skopus-Strukturen, bei denen der Operator entweder dem Skopus vorangestellt ist oder in insertierter Stellung verwendet wird. Nur bei diesen Stellungsvarianten erscheint uns die projektive Kraft des Operators unzweifelhaft gegeben. In nachgestellter Position dagegen 
ist die Projektionskraft des Operators umstritten (cf. dazu Schneider/Butterworth/Hahn 2018: 158). Das heißt, im Unterschied zu Barden/Elstermann/Fiehler (cf. 2001) schließen wir Operatoren, die dem Skopus nachgestellt werden, nicht in unser Konzept mit ein. Sie können weder einen vorgreifenden Verstehenshinweis liefern noch eine Leerstelle eröffnen.

Die rückwärts gerichtete Relationierung unterscheidet sich in mehrerer Hinsicht von der vorwärts gerichteten. Während der Operator nach vorn hin eine Leerstelle eröffnet, die nach Auffüllung verlangt, ist die rückwärts gerichtete Bindung von anderer Art. Auch die Reichweite ist verschieden. [...] Auch wenn der Operator oft direkt auf das zuvor Gesagte Bezug nimmt, handelt es sich bei der rückwärts gerichteten Relationierung nicht um eine Verstehensanweisung der Art, wie sie für das Verhältnis von Operator zum Skopus gilt. Die Relation nach hinten ist zunächst allgemein als konnektierende Funktion des Operators aufzufassen.

(Fiehler et al. 2004: 279)

Da nachgestellte Operatoren eine andere Funktion und damit eine andere Verwendungsart besitzen, weicht ihr Status ebenso ab, weshalb sie nach unserem Verständnis gesondert zu betrachten sind. Demzufolge werden Operatoren in dieser Positionierung in der weiteren Analyse nicht berücksichtigt.

Entsprechend unserer Einteilung gemäß funktionalen, interaktiven Einheiten in der gesprochenen Sprache in Opposition zum Satzbegriff und weil wir auch die insertierte und nicht nur vorausgehende Stellung des Operators berücksichtigen, verzichten wir außerdem auf die terminologische Positionsbeschreibung „Vorvorfeld“. Auch wenn diese Bezeichnung eingeführt wurde, um Voranstellungen in der Syntax gesprochener Sprache zu erfassen (cf. z. B. Auer 1997), ist sie nicht unumstritten (cf. Schröder 2006), denn dieses Konzept beruht trotz allem auf dem schriftsprachlichen Verbalsatz als Orientierungsgröße und wird dem written language bias folgend auf mündlichen Sprachgebrauch übertragen. Darüber hinaus wird durch die Verortung des Operators im vermeintlichen Vorvorfeld des Skopus dem nicht Rechnung getragen, dass die projektive Kraft des Operators ja über mehrere Einheiten hinweg wirken kann (cf. Schröder 2006: 227). Aus diesen genannten Gründen nehmen wir die Positionsbeschreibung „Vorvorfeld“ als definitorisches Kriterium von unserem Strukturkonzept aus.

Diese Ausführungen zu Diskursmarkern im Allgemeinen und Operator-Skopus-Strukturen im Speziellen machen noch einmal deutlich, weshalb die Perspektive auf einen interaktiven Äußerungs- und Einheitenbegriff gelenkt wurde. Im Fokus steht die pragmatische diskursorganisierende Funktionsweise dieser sprachlichen Strukturen, wobei es immer gilt, einen breiten Äußerungskontext sowie den dynamischen Charakter der Sprache in der Interaktion zu berücksichtigen. Daher wird funktionalen, interaktiven Einheiten gegenüber Sätzen oder syntaktischen Strukturen der Vorzug gegeben. Wie sich das nun konkret in der Interaktion beobachten und analysieren lässt, soll im folgenden Kapitel dargestellt werden.

\section{$3 \quad$ Korpus und Daten}

Unsere Datenbasis besteht aus Aufnahmen gesprochener Sprache in Form von Interviews und Freundesgesprächen des Stadtsprachenkorpus (Urban Language Austria - im Folgenden ULAKorpus genannt). ${ }^{1}$ Die Aufnahmen wurden im Rahmen des Forschungsprojekts „Wien und

\footnotetext{
${ }^{1}$ Dieses Korpus ist momentan nur projektintern verfügbar, soll aber zukünftig online veröffentlicht werden.
} 
Graz - Städte und ihre sprachlichen Strahlkräfte“ durchgeführt. ${ }^{2}$ Es handelt sich dabei um 93 Gesprächsaufnahmen von 73 SprecherInnen aus den Großräumen Wien und Graz, mit einer transkribierten Gesamtdauer von knapp 62 Stunden. Die SprecherInnen sind zwei Altersgruppen zuzuordnen: Die erste Gruppe bilden junge SprecherInnen im Alter zwischen 20 und 30 Jahren und die zweite Gruppe besteht aus SprecherInnen über 65 Jahre. Die SprecherInnen lassen sich in folgende Gruppen einteilen (siehe Tabelle $1^{3}$ ):

\begin{tabular}{|c|c|c|c|c|c|}
\hline \multicolumn{2}{|c|}{ Alter } & \multicolumn{2}{c|}{ Geschlecht } & \multicolumn{2}{c|}{ Ort } \\
\hline $20-30$ & $>65$ & Männlich & Weiblich & Graz & Wien \\
\hline 22 & 51 & 33 & 40 & 47 & 26 \\
\hline
\end{tabular}

Tabelle 1: Probandenspezifika

Die Gespräche wurden mittels EXMARaLDA weiterverarbeitet: In einem ersten Schritt wurden nach geringfügig modifizierten GAT 2-Konventionen Basistranskripte erstellt. ${ }^{4}$ Dann wurde aus allen Transkripten mittels des EXMARaLDA-Corpus-Managers COMA ein Gesamtkorpus generiert, das in weiterer Folge mit dem Such- und Analysewerkzeug EXAKT ausgewertet werden konnte. Das Korpus wurde für unsere Zwecke nach unterschiedlichen OperatorSkopus-Strukturen abgefragt und eine Liste der vorkommenden Operatoren in den Gesprächen erstellt. Aus diesen wurden dann repräsentative Strukturen mit erweitertem Äußerungskontext ausgewählt, die diskursanalytisch ausgewertet wurden.

\subsection{Datenerhebung}

Für die Analyse werden, wie zuvor erwähnt, Interviews $(n=73)$ und Freundesgespräche $(n=$ 20) urbaner SprecherInnen aus dem Raum Graz und dem Raum Wien des ULA-Korpus herangezogen. Im formellen Setting des Interviews kennen sich die beiden SprecherInnen (Exploratorin und Gewährsperson) nicht. Die Exploratorin bemüht sich im Gespräch um einen höflichdistanzierten Umgang und eine möglichst standardnahe Sprechweise. In dieser Form des soziolinguistischen Interviews werden Fragen zum privaten wie beruflichen Werdegang, zu Sprachbiographie sowie Sprachgebrauch, Spracheinstellung und historischem Sprachwissen gestellt.

Am informellen Freundesgespräch nehmen jeweils zwei Personen teil, die einander gut kennen (entweder als FreundInnen, gute Bekannte oder (Ehe-)PartnerInnen). Für dieses Setting gibt es keine Vorgaben. Die SprecherInnen sollen einen ungezwungenen Dialog führen, um ihren natürlichen Sprachgebrauch erheben zu können. Es wird jedoch ein Angebot an Themenkärtchen bereitgestellt, das fakultativ verwendet werden darf, z. B. als Icebreaker zu Beginn des Gesprächs, sollte das Gespräch ins Stocken geraten oder einfach nur Interesse an den Themenimpulsen bestehen. Es handelt sich dabei um alltägliche Themen wie Hobbys, Sport, Filme oder

\footnotetext{
${ }^{2}$ Dieses Projekt ist Teil des vom FWF geförderten Spezialforschungsbereichs „Deutsch in Österreich“ (FWF, F 60).

${ }^{3}$ Die Datengrundlage wurde für die vorliegende Analyse nicht normalisiert, da die außersprachlichen Faktoren keinen beobachtbaren Einfluss auf die Gebrauchs- bzw. Funktionsweisen der von uns untersuchten Operatoren aufweisen (siehe Kapitel 4).

${ }^{4}$ EXMARaLDA ist eine frei verfügbare Software mit unterschiedlichen Tools zur Aufbereitung von Gesprächsdateien. Zum gesprächsanalytischen Transkriptionssystem (GAT 2) und zu den Konventionen für das Basistranskript siehe Selting et al. (2009).
} 
um Gedankenspiele wie einen Lottogewinn machen, auswandern oder einen Wunsch frei haben.

\subsection{Datenanalyse und Interpretation}

Für die Analyse der Operatoren des kommunikativen Status wurde im Korpus nach mehreren Strukturen gesucht. Folgende Lexeme und kurze Phrasen wurden dafür abgefragt:

ehrlich/offen/unter uns/im Vertrauen (gesagt), bei aller/allem Liebe/Respekt, wirklich, tatsächlich, Tatsache ist, in der Tat, eindeutig, im Ernst, ernsthaft, Spaß beiseite, (im) Scherz, sicher(lich), ganz sicher, wichtig(er), vor allen Dingen, vor allem, zweifellos, echt, garantiert, fest steht, hundertprozentig, unvermeidlich, primär, wahrhaftig.

Davon sind frequente Treffer insbesondere für die Lexeme beziehungsweise Phrasen ehrlich $(\mathrm{n}=34)$, wirklich $(\mathrm{n}=28)$, sicher $(\mathrm{n}=15)$ und vor allem $(\mathrm{n}=34)$ zu verzeichnen.

Für die Analyse der Operatoren des mentalen Status wurde im ULA-Korpus gesucht nach:

ich meine, ich finde, ich weiß, ich glaube, ich würde sagen, grob geschätzt, nur eine Idee.

Unter ihnen sind vorherrschend: ich meine $(\mathrm{n}=672)$, ich finde $(\mathrm{n}=153)$, ich wei $\beta(\mathrm{n}=58)$ und ich glaube $(\mathrm{n}=281)$.

In der Gegenüberstellung der frequenten Strukturen (siehe Tabelle 2), in denen die angeführten Lexeme/Phrasen mit oder ohne Operatorfunktion in Erscheinung treten, lassen sich folgende Verteilungen beobachten:

\begin{tabular}{|l|r|r|}
\hline & ohne Operatorfunktion & mit Operatorfunktion \\
\hline ehrlich & $54(61 \%)$ & $34(39 \%)$ \\
\hline wirklich & $710(96 \%)$ & $28(4 \%)$ \\
\hline sicher & $565(97 \%)$ & $15(3 \%)$ \\
\hline vor allem & $172(84 \%)$ & $33(16 \%)$ \\
\hline ich meine & $5(1 \%)$ & $672(99 \%)$ \\
\hline ich finde & $6(4 \%)$ & $153(96 \%)$ \\
\hline ich wei $\beta$ & $5(8 \%)$ & $58(92 \%)$ \\
\hline ich glaube & $100(26 \%)$ & $281(74 \%)$ \\
\hline total & $1617(56 \%)$ & $1274(44 \%)$ \\
\hline
\end{tabular}

Tabelle 2: Verteilung von Lexemen/Phrasen mit und ohne Operatorfunktion

Besonders auffällig in Tabelle 2 verhalten sich ich meine, ich finde, ich wei $\beta$, da ihr Gebrauch eindeutig in Operatorfunktion überwiegt. Vergleichsweise selten hingegen werden wirklich und sicher als Operator verwendet.

Alle ausgewerteten Operator-Skopus-Strukturen erfüllen unsere in Kapitel 2.2 vordefinierten Kriterien. Das heißt, sie sind jeweils nicht vollständig in die Äußerung integriert, sondern von ihr abgesetzt. Allein können sie jedoch nicht stehen, denn sie erfüllen nicht die Funktion einer selbständigen interaktiven Einheit. Vielmehr eröffnen sie in ihrer Position eine Leerstelle, die nach Auffüllung durch den Skopus verlangt. Gleichzeitig besitzen sie dadurch auch projektive Kraft auf die nachfolgende Äußerung. Operator und Skopus bilden letztlich eine zweigliedrige, asyndetische Struktur und erst in ihrer Kombination entsteht eine semantisch und syntaktisch 
vollständige Äußerung. Es sollen nun einige ausgewählte Operator-Skopus-Strukturen mit Hilfe von Transkriptausschnitten exemplarisch diskutiert und analysiert werden. Vorangestellt wird den Beispielen jeweils eine Übersicht der Gebrauchsfrequenzen innerhalb der SprecherInnengruppen. ${ }^{5}$

Ziel der Analyse ist es, zu zeigen, wie der Einsatz von Operatoren bzw. Operator-Skopus-Strukturen durch ihren Gebrauch in unterschiedlichen Gesprächssituationen und im Rahmen der gegebenen außersprachlichen Faktoren stattfindet, aus pragmatischer Sicht erklärt und im Zuge dessen interpretiert werden kann.

\subsubsection{Operatoren zur Charakterisierung des kommunikativen Status}

Um den Stellenwert beziehungsweise die Qualität einer Äußerung zu verdeutlichen, kann sich der Sprecher/die Sprecherin Operatoren bedienen, die die Geltung, die Relevanz, die Offenheit oder die Modalität von Äußerungen charakterisieren. Die Geltung kann zum Beispiel in Bezug auf den Wahrheitsgehalt einer Äußerung oder deren Sicherheit ausgedrückt werden. Wir wollen nun tendenzielle Verwendungsweisen der dargestellten Operatoren unseres Korpus aufzeigen. Die folgenden Gesprächsauszüge sollen das illustrieren. Es handelt sich dabei um exemplarisch ausgewählte Operator-Skopus-Strukturen, wie sie in ähnlicher Form häufiger in unserem Korpus vorkommen. Die einzelnen Beispiele stehen also stellvertretend für die größere Menge an Operator-Belegen.

\section{Beispiel 1) wirklich}

Als erstes Beispiel soll der Einsatz von wirklich in Operatorfunktion beschrieben werden. Das Vorkommen dieses Operators zeigt sich geordnet nach den Parametern Alter und Geschlecht (siehe Tabelle 3) in unserem Korpus folgendermaßen verteilt:

\begin{tabular}{|l|r|r|r|}
\hline \multicolumn{5}{|c|}{ wirklich } \\
\hline & alt & jung & Gesamtanzahl \\
\hline männlich & 7 & 6 & $n=13$ \\
\hline weiblich & 7 & 8 & $n=15$ \\
\hline total & 14 & 14 & $n=28$ \\
\hline
\end{tabular}

Tabelle 3: Verteilung von wirklich nach Alter und Geschlecht

Auch wenn es sich hierbei um kein hochfrequentes Vorkommen des Operators handelt, zeigt die Tabelle, dass SprecherInnen beider Alters- oder Geschlechtsgruppen Gebrauch von Operator-Skopus-Strukturen mit wirklich machen. Um nun die Einbettung in den Gesprächskontext zu illustrieren, folgt im Weiteren ein Ausschnitt aus einem Freundesgespräch zweier junger Studierender aus Graz. Thema ist die Umsetzung eines automatisch erstellten Inhaltsverzeichnisses im Textprogramm Word. Sprecher 4073 erzählt, von der Betreuerin seiner Bachelorarbeit dafür Anleitung und Tipps bekommen zu haben, und seine Gesprächspartnerin Sprecherin

\footnotetext{
${ }^{5}$ Es werden dabei die Vorkommen in absoluten Zahlen angegeben, da die Ermittlung relativer Häufigkeiten das Bestehen funktionsäquivalenter Varianten voraussetzen würde, welche miteinander in Relation gesetzt werden könnten. Im Falle der Operatoren lässt sich aber auf diese Weise keine Referenzmenge bilden. Daher und auch aufgrund individueller Präferenzen unter den SprecherInnen sind die Gebrauchsfrequenzen nicht zu vergleichen.
} 
4072 bittet ihn, sein Wissen mit ihr zu teilen. ${ }^{6}$ Sie selber scheitere immer an dieser Funktion, weshalb sie im Gespräch ihrer Verzweiflung und Hilfsbedürftigkeit verstärkend Ausdruck verleiht, indem sie wirklich als Operator verwendet.

[1]

\begin{tabular}{|c|c|c|}
\hline & $531[13: 01.9]$ & $532[13: 04.0]$ \\
\hline $4073[v]$ & $=$ hauptsächlich wir ham gar nicht so viel: BAKKarbeit gredet; & $=$ so a paar FRAgen \\
\hline
\end{tabular}

[2]

$533^{[13: 05.1]}$
4073 [v] waren halt- (-) dann is mir nur $\mathrm{x}$ ein zwei tipps bei $<<$ englische Aussprache $>$

[3]

\begin{tabular}{|c|c|c|c|}
\hline & $534[13: 07.4] 535[13: 08.1] 5$ & $7] 537$ [13:09.0] & $538[13: 09.7]$ \\
\hline $\begin{array}{l}4073[\mathrm{v}] \\
4072[\mathrm{v}]\end{array}$ & WORD> geben, (--) was SUper is, & $(-)$ ah SO & wie ma INhaltsverzeichnis \\
\hline
\end{tabular}

[4]

\begin{tabular}{|lccc|}
\multicolumn{1}{c}{$539[13: 11.4] 540[13: 11.6] 541[13: 12.0]$} & $542[13: 12.9] 543[13: 13.0] 544[13: 13.4]$ \\
\hline $\mathbf{4 0 7 3}[\mathbf{v}]$ & gscheit erstellt; & ja MACH i; & \\
$\mathbf{4 0 7 2}$ [v] & HA:, & $=$ kannst_ma des $<<$ kichernd $>$ ZEIgen; $>$ & weißt \\
\hline
\end{tabular}

[5]

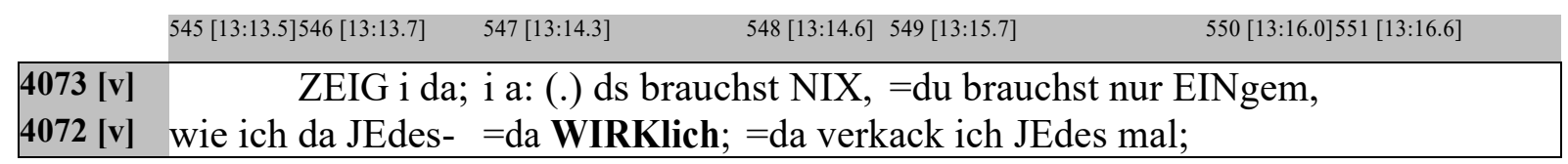

[6]

4073 [v] =INhaltsverzeichnis erstellen, (--) dann markierst du die Überschriften, =und die

[7]

$$
554 \text { [13:21.3] } 555 \text { [13:22.9]556 [13:23.1] }
$$

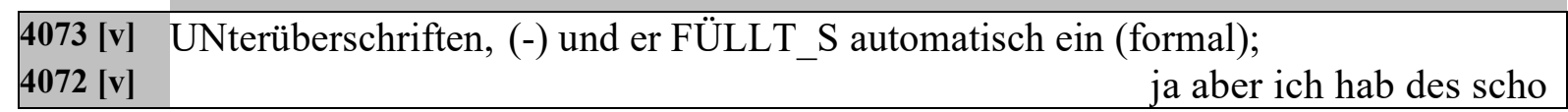

[8]

\begin{tabular}{|ccc|}
\multicolumn{1}{l|}{$557[13: 23.6] \quad 558[13: 24.1] 559[13: 24.3]$} & $560[13: 25.2]$ \\
\hline $\mathbf{4 0 7 3}[\mathbf{v}]$ & und IMmer wenn- & JA, \\
$\mathbf{4 0 7 2}[\mathbf{v}]$ & mal proBIERT, =ich hab des noch NIE zammkriegt; \\
\hline
\end{tabular}

(ULA-Korpus, 4072_GRAW_jung_w_CAF, Seg. 539-560) ${ }^{7}$

Mithilfe des Einsatzes des Operators wirklich verdeutlicht die Sprecherin 4072, dass sie ernsthaft auf Hilfe angewiesen ist und sich Unterstützung von ihrem Gegenüber wünscht, da sie sich

\footnotetext{
6 Die Nummerierung der SprecherInnen dient der Anonymisierung und wird gleichzeitig als ProbandInnensigle verwendet.

${ }^{7}$ Das Zitat aus dem ULA-Korpus lässt sich wie folgt verstehen: Aufnahme bzw. ProbandInnenkürzel (z. B. 4072), Erhebungsort (z. B. Graz Waltendorf = GRAW), Altersgruppe (jung/alt), Geschlecht der Gewährsperson (w/m), Setting (Conversation among friends $=\mathrm{CAF} /$ Analytical interview $=\mathrm{AI}$ ) und die fortlaufende Nummerierung der zitierten Segmente (Seg. $\mathrm{x}-\mathrm{y}$ ). Im Folgenden werden alle Gesprächsausschnitte nach diesem Muster zitiert.
} 
nicht in der Lage fühlt, allein das Problem zu lösen, wie im Skopus der Äußerung ausgeführt wird. Wirklich ist also ein Signal der Offenheit und soll gleichermaßen den Wahrheitsgehalt der Äußerung ausdrücken. Auffällig ist in diesem Beispiel, dass auf dem insertierten Operator der Fokusakzent der Intonationsphrase liegt, was die prosodische Absetzung bewirkt. Es folgt eine leicht fallende Tonhöhenbewegung nach dem Operator, bevor der Skopus folgt. Der Operator verleiht damit gleichzeitig der ausgedrückten Bitte „kannst ma des ZEIgen“ Nachdruck und veranlasst Sprecher 4073 dazu, gleich zu erklären, wie man bei der Erstellung eines Inhaltsverzeichnisses in Word vorgeht. Dieses Beispiel zeigt außerdem, dass der Operator nicht nur eine einzelne pragmatische Funktion erfüllt. Vielmehr transportiert er ein Bündel an Funktionsweisen und reichert damit den Äußerungsgehalt der Aussage an. Es gelingt Sprecherin 4072 also, mittels des Operators gleich auf mehrere Aspekte ihrer Sprecherintention zu verweisen.

\section{Beispiel 2) sicher}

Tabelle 4 bietet eine Übersicht über die Verteilung des zweiten von uns analysierten Operators, sicher, nach Alter und Geschlecht:

\begin{tabular}{|l|r|r|r|}
\hline \multicolumn{4}{|c|}{ sicher } \\
\hline & alt & jung & Gesamtanzahl \\
\hline männlich & 8 & 0 & $n=8$ \\
\hline weiblich & 6 & 1 & $n=7$ \\
\hline total & 14 & 1 & $n=15$ \\
\hline
\end{tabular}

Tabelle 4: Verteilung von sicher nach Alter und Geschlecht

Auch wenn sicher nur ab und an in unserem Korpus Verwendung findet, sind die Belege vorrangig unter den älteren SprecherInnen auszumachen. Daher soll ein Ausschnitt eines Freundesgesprächs zweier Pensionisten aus der Grazer Umlandgemeinde Thal ins Feld geführt werden, in welchem sicher in Operatorfunktion Gebrauch findet. Die beiden Gesprächspartner sprechen über die Geschichte und Entwicklung ihres Wohnorts, Veränderungen und gesellschaftliche Umstrukturierungen. Sprecher 4022 ruft sich mit seinem Gesprächspartner in Erinnerung, welch zahlreiche Geschäfte und Gasthäuser es ursprünglich im Ort gab, die nach und nach verschwunden sind. Auch der Thaler See, ein beliebtes Ausflugsziel der Grazer, hat sich in ihren Augen stark gewandelt. Sicher als Operator wird von Sprecher 4022 verwendet um zu signalisieren, dass er keinen Zweifel an seinem vorgebrachten Einwand in der Äußerung im Skopus hat.

[1]

$1338[32: 59.2]$
4022_F [v] und (-) und (.) du muasst denken des (.) wor ja (-) äh [thol]O äh (.) da [thaler

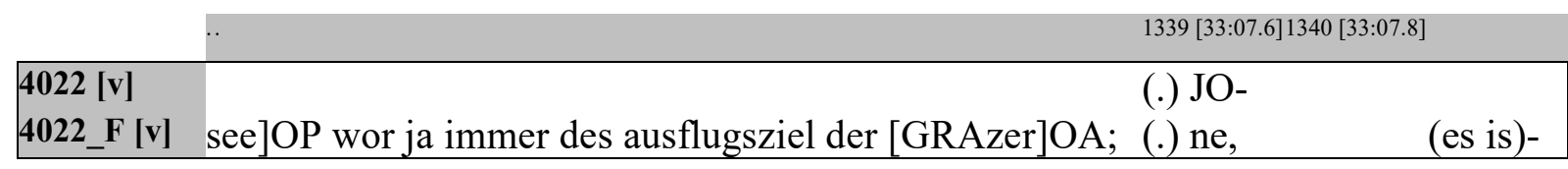


$[3]$

\begin{aligned} \multicolumn{1}{l}{$1342[33: 08.7]$} & $1343[33: 10.8] 1344[33: 10.9] \\$\hline 4022_F [v] & $($.$) i maan (-)$ es is HEIT aa no; $=$ ne, $\quad=$ ober $(--)$ ober in net mehr in den \end{aligned}

[4]

\begin{tabular}{|lll|}
\multicolumn{1}{l}{$1345[33: 14.4] 1346[33: 14.6]$} & \multicolumn{1}{c|}{$1347[33: 15.1] 1348[33: 16.8]$} \\
\hline 4022 [v] & & na JO; \\
4022_F [v] & RAHmen wos wor; $=$ ne, & $(-)$ früher wor jo da BAdebetrieb- $(-)$ do woren on om \\
\hline
\end{tabular}

[5]

\begin{tabular}{|ll|}
\multicolumn{1}{|l|}{$1349[33: 20.2] 1350[33: 20.4]$} \\
\hline 4022 [v] & $(-)$ äh fünftausend LEIT ouft; $=$ ne, $\quad \begin{array}{l}\text { (eh do) am meisten woren } \\
(-) \text { ja- }\end{array}$ \\
\hline
\end{tabular}

$[6]$

\begin{tabular}{|llll|}
\multicolumn{1}{l}{$1351[33: 21.0]$} & $1352[33: 22.6]$ & $1353[33: 23.5] 1354[33: 24.0] 1355[33: 24.7]$ \\
\hline 4022 [v] & ZEHNtausend amol; & ober äh des is- & $(-)$ sicher die leit stöllen holt \\
4022_F [v] & (.) jojo JO; & aiso- & \\
\hline
\end{tabular}

[7]

$1356[33: 27.8] 1357[33: 29.1] 1358[33: 29.6]$

\begin{tabular}{l}
$\begin{array}{l}\text { 4022 [v] } \\
\text { 4022_F [v] }\end{array}$ \\
\hline
\end{tabular}

[8]

\begin{tabular}{|c|c|c|c|}
\hline & 1359 [33:32.0] & $1360[33: 33.5] 1361[33: 34.6]$ & $1362[33: 35.0] 1363[33: 35.5$ \\
\hline $4022[\mathrm{v}]$ & der sogt & n & \\
\hline
\end{tabular}

(ULA-Korpus, 4022_THAL_alt_m_CAF, Seg. 1345-1363)

Sicher wird äußerungsinitial gebraucht und ist prosodisch durch die Pause (Dauer 0.25s) von der vorhergehenden Äußerung abgesetzt. Der Operator nimmt im vorliegenden Gesprächsausschnitt vordergründig die Funktion einer Einräumung mit relativierendem Charakter ein. Sprecher 4022 macht mit dem eingesetzten Operator sein Verständnis für die veränderte Nutzung des Naherholungsgebiets deutlich, die er dem im Skopus der Äußerung erläuterten gesellschaftlichen Wandel und den wachsenden Ansprüchen der Menschen zuschreibt. Die rückläufige Anzahl an Badegästen erkläre sich durch die zunehmend größer werdende Erwartungshaltung gegenüber der Beschaffenheit beziehungsweise Sauberkeit des Sees, weshalb sich die BesucherInnen heute mehr als früher überlegen würden, ob sie darin schwimmen wollen oder nicht. Sprecher 4022 drückt mit seiner Aussage auch seine Zustimmung in Bezug auf die Ausführungen seines Freundes Sprecher 4022_F aus, da dieser vorausgehend darauf hinweist, dass die Besucherzahlen in der Region rückläufig sind. Sprecher 4022 unterstützt also dessen Ansicht, indem er einräumt, dass die Ansprüche der Menschen (z. B. die Wasserqualität des Sees betreffend) ausschlaggebendes Kriterium für die rückläufige Frequentierung des Thaler Sees sind.

\section{Beispiel 3) ehrlich}

Für ehrlich in Operatorfunktion zeigen sich sortiert nach Alter und Geschlecht folgende Verwendungen (siehe Tabelle 5): 


\begin{tabular}{|l|r|r|r|}
\hline \multicolumn{4}{|c|}{ ehrlich } \\
\hline & alt & jung & Gesamtanzahl \\
\hline männlich & 12 & 4 & $n=16$ \\
\hline weiblich & 7 & 11 & $n=18$ \\
\hline total & 19 & 15 & $n=34$ \\
\hline
\end{tabular}

Tabelle 5: Verteilung von ehrlich nach Alter und Geschlecht

Der Operator entfällt in unserem Korpus auf alle SprecherInnen in jeder Alters- und Geschlechtsgruppe. Bei folgendem Gesprächsausschnitt handelt es sich um eine Äußerungssequenz aus einem Freundesgespräch zweier junger Gewährspersonen (eine junge Frau, ein junger Mann) aus Graz. Die Freunde unterhalten sich in ungezwungenem Rahmen über Themen ihrer Wahl, es steht ihnen aber auch ein Angebot an Themenkärtchen zur Verfügung, derer sie sich bedienen dürfen. Am ausgewählten Punkt des Gesprächs haben sie soeben ein Kärtchen ausgewählt, auf dem ,über Nacht berühmt werden” steht. Es soll sich dabei um ein Gedankenspiel handeln, zu dem beide frei assoziieren.

[1]

\begin{tabular}{|c|c|c|c|}
\hline & $1583[29: 33.8] 1584[29: 34.3]$ & $1585[29: 35.1] 1586[29: 35.6] 1587[29: 35.8] 1588[29: 36.2]$ & $1589[29: 37.0]$ \\
\hline $\begin{array}{l}4076[v] \\
4077[v]\end{array}$ & $\begin{array}{l}\text { UNterrichtsminist } \\
\text { da bist du }\end{array}$ & du bist- $=$ wirst in & $\begin{array}{l}\text { außer ma haßt } \\
\text { wem wem }\end{array}$ \\
\hline
\end{tabular}

[2]

\begin{tabular}{|c|c|c|c|}
\hline & & & \\
\hline $\begin{array}{l}40 / 0[v] \\
4077[v]\end{array}$ & $\begin{array}{l}\text { angela MERkel; } \\
\text { geHASST; }\end{array}$ & $((\text { kichert }))^{((\text {kichert }))}$ & (-) dann hängt ma im POster- \\
\hline
\end{tabular}

[3]

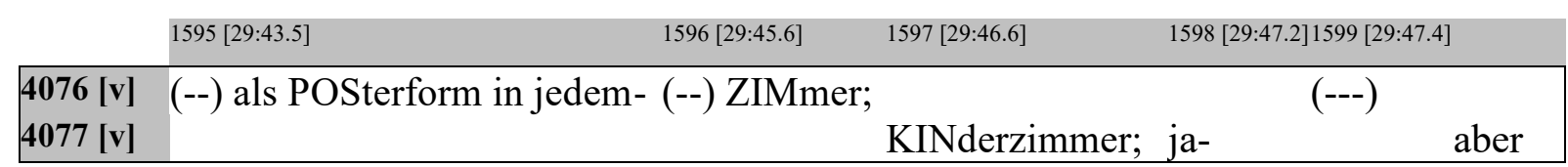

[4]

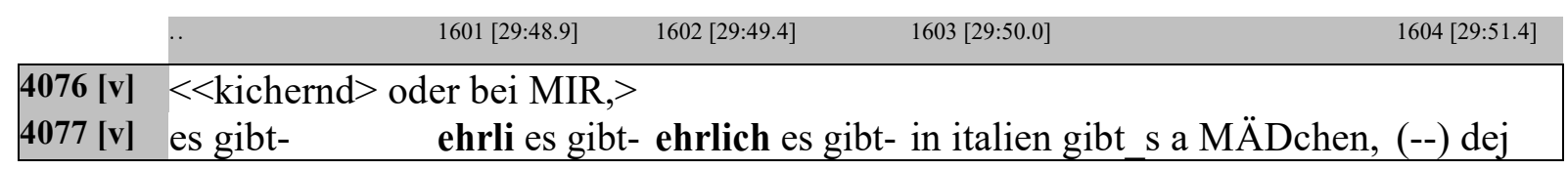

[5]

$$
1605 \text { [29:53.2] } 1606 \text { [29:54.4] } 1607 \text { [29:55.2] }
$$

4077 [v] steht auf DEUTSCHland, und des hat sich SELwer- hat die HAAre- an gleichen $[6]$

\begin{tabular}{|c|c|c|}
\hline & $1608[29: 57.2]$ & 1609 [29:59.2] 1610 [29:59.7] \\
\hline $\begin{array}{l}4076[v] \\
4077[v]\end{array}$ & (--) und & DES find i cool, \\
\hline
\end{tabular}

\begin{tabular}{|cc|}
\multicolumn{1}{c|}{$1611[30: 00.0] 1612[30: 00.6]$} & $1613[30: 01.3]$ \\
\hline $\mathbf{4 0 7 6}[\mathbf{v}]$ & GFALLT ma; \\
$\mathbf{4 0 7 7}[\mathbf{v}]$ & $(-)$ die steht voll auf DEUTSCHland, \\
\hline
\end{tabular}

(ULA-Korpus, 4077_WEINITZ_jung_m_CAF, Seg. 1583-1609) 
In obigem Ausschnitt überlegen die Freunde, welche Berühmtheit sie sein möchten oder eben nicht und aus welchen Gründen, bis sie zum Berufsfeld PolitikerIn gelangen. An diesem Punkt setzt der Gesprächsausschnitt ein. Zuerst wird darüber gesprochen, dass man als PolitikerIn ein durchaus streitbares Image hat und auch Hassgefühle entgegengebracht bekommen kann. Dann konzentriert sich das Gespräch auf die deutsche Bundeskanzlerin Angela Merkel. Sprecher 4076 gibt sich als Fan zu erkennen und seine Gesprächspartnerin erzählt von einer Italienerin, die ihr Äußeres (Haarschnitt und Kleidung) an die Politikerin angleicht. Um diese besondere Art der Verehrung durch Imitation glaubhaft zu machen, verweist die Sprecherin 4077 wiederholt durch äußerungsinitiales "ehrli es gibt- ehrlich es gibt" (die Wiederholung beruht auf dem Overlap der beiden Sprecher) auf den Wahrheitsgehalt ihrer Äußerung im Skopus. Da die Sprecherin über die Tatsache einer solchen Anhängerschaft sehr erstaunt ist, erscheint es ihr notwendig, die Verlässlichkeit ihrer Aussage zu unterstreichen und sie bedient sich daher des Operators ehrlich. Er dient dazu, Sprecher 4076 zu versichern, dass Sprecherin 4077 die Wahrheit sagt beziehungsweise von der Richtigkeit ihrer Information überzeugt ist. Die Äußerung wird mit dem Operator ehrlich eingeleitet und entwickelt durch die vorgreifende Verdeutlichung, wie die nachfolgende Äußerung im Skopus aufgefasst und interpretiert werden soll, ihre projektive Kraft. Sprecherin 4077 scheint mit dem Einsatz des Operators bezwecken zu wollen, dass etwaige Vorbehalte abgebaut werden und ihr Gesprächspartner keine Zweifel an der Verlässlichkeit ihrer Aussage hegt. Dem Ganzen geht dabei keine Äußerung voraus, die eine unbedingte Notwendigkeit des Einsatzes des Operators erfordert, doch erfüllt ehrlich eine zentrale Funktion: Es dient zur Unterstützung der Geltung der Aussage.

\section{Beispiel 4) vor allem}

In der folgenden tabellarischen Aufstellung (siehe Tabelle 6) sind die Verwendungshäufigkeiten von vor allem in Operatorfunktion gemäß den Variablen Alter und Geschlecht abgebildet:

\begin{tabular}{|l|r|r|r|}
\hline \multicolumn{4}{|c|}{ vor allem } \\
\hline & alt & jung & Gesamtanzahl \\
\hline männlich & 7 & 8 & $n=15$ \\
\hline weiblich & 4 & 14 & $n=18$ \\
\hline total & 11 & 22 & $n=33$ \\
\hline
\end{tabular}

Tabelle 6: Verteilung von vor allem nach Alter und Geschlecht

Der Operator zeigt sich zwar nicht ausgenommen frequent, aber bei SprecherInnen jeder Altersund Geschlechtsgruppe unseres Korpus. Nachfolgendes Beispiel stammt aus einem Freundesgesprächs eines jungen Ehepaars aus Gerasdorf (Niederösterreich). Es wird über den bereitgestellten Themenimpuls „Gedanken lesen“ gesprochen und welche negativen Implikaturen diese Fähigkeit hätte. Sowohl Sprecher 4057 als auch Sprecherin 4067 geben ihrer ablehnenden Haltung gegenüber der Möglichkeit, Gedanken zu lesen, Ausdruck. Sie führen dazu die sozialen Probleme oder Spannungen an, die sich daraus ergeben könnten.

[1]

1339 [36:00.9] 1340 [36:04.6]

$1341[36: 06.1]$

4057 [v] aiso (---) ma denkt si ganz geNAU wos, und sagt_s net GANZ genau so, =sondern 
[2]

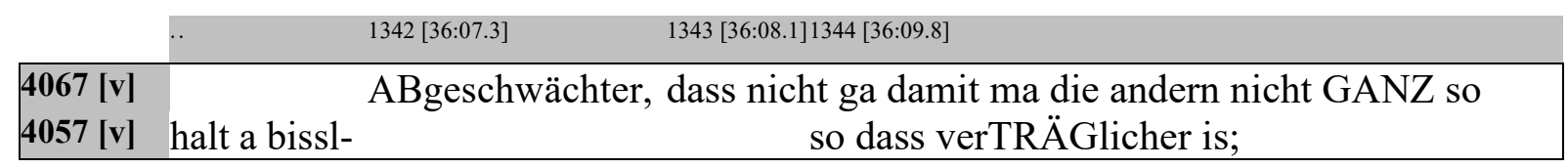

[3]

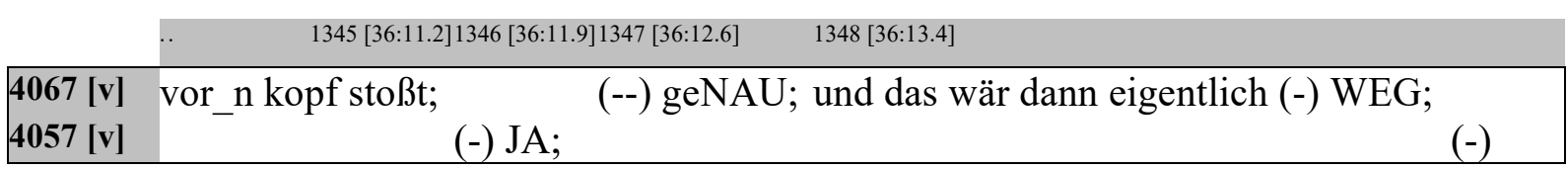

[4]

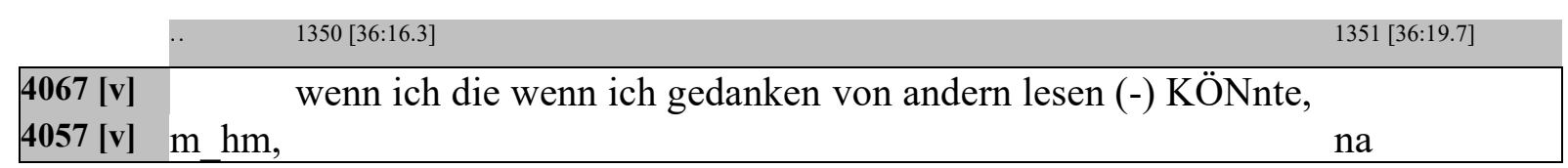

[5]

\begin{tabular}{|lll}
\multicolumn{1}{l}{$1352[36: 20.8] 1353[36: 21.7]$} & $1354[36: 22.9]$ \\
\hline $4067[\mathbf{v}]$ & das is- ich ich will nicht WISsen; vor allem (---) da erste gedanke is \\
$\mathbf{4 0 5 7}[\mathbf{v}]$ & FURCHTbar;
\end{tabular}

[6]

\begin{tabular}{|c|c|c|}
\hline & & $1355[36: 26.9] 1356[36: 27.5] 1357[36: 29.8]$ \\
\hline $\begin{array}{l}4067[v] \\
4057[v]\end{array}$ & ja oft auch gar nicht so GMEINT; & $\begin{array}{l}\text { und nicht so: net (--) so im exTREmen } \\
\text { JA; } \\
\text { gar net so GUT ja; }\end{array}$ \\
\hline
\end{tabular}

[7]

\begin{tabular}{|lll}
$1358[36: 31.0] 1359[36: 31.8]$ & \multicolumn{1}{c}{$1360[36: 33.5]$} \\
\hline 4067 [v] & gmeint; $(-)$ sondern eher- (1.6) es is halt einfach was was da ei was was da grad (-)
\end{tabular}
[8]

\begin{tabular}{|llll|}
\multicolumn{1}{c}{$1361[36: 38.5] 1362[36: 39.2] 1363[36: 41.0]$} & $1364[36: 41.7]$ \\
\hline $4067[\mathbf{v}]$ & durch_n KOPF schießt, und- & und was ma- \\
$\mathbf{4 0 5 7}[\mathbf{v}]$ & (.) JA; & $(--)$ un was ma selber dann wiede:r reviDIERT; \\
\hline
\end{tabular}

[9]

\begin{tabular}{|c|c|}
\hline & $1365[36: 42.0] 1366[36: 42.4]$ \\
\hline $\begin{array}{l}4067[v] \\
4057[v]\end{array}$ & geNAU; \\
\hline
\end{tabular}

(ULA_Korpus, 4057_GERAS_jung_m_CAF, Seg. 1339-1366)

Sprecher 4057 weist darauf hin, dass das, was man sich denkt, oft im Zuge eines respekt- und taktvollen Umgangs im sozialen Miteinander modifiziert verbalisiert wird. Unreflektiert geäußert könnten die Gedanken das Gegenüber vor den Kopf stoßen. Sprecherin 4067 ergänzt zustimmend, dass Gedanken oft nur erste, bedingt steuerbare Impulse seien. An dieser Stelle setzt sie den Operator vor allem ein, um klarzustellen, dass initiale Gedanken oft nicht mit einer differenzierten Meinung gleichzusetzen seien. Diese Aussage scheint für sie von großer Bedeutung zu sein beziehungsweise großes Gewicht in ihrer Argumentation, warum man nicht Gedanken lesen können sollte, zu besitzen. Dazu trägt auch der formale Rahmen der äußerungsinitialen Position des Operators mit nachfolgender längerer Pause (Dauer 0.75s) bei. Durch diese Form der Verwendung von vor allem verleiht die Sprecherin so der angeschlossenen 
Aussage im Skopus Nachdruck und unterstreicht damit ihre Wichtigkeit. Dadurch, dass der Operator mit einer Sprechpause abgesetzt, also nicht nur syntaktisch, sondern auch prosodisch markiert ist, wird eine Erwartungshaltung aufgebaut, die die Relevanz der Äußerung verdeutlichen soll. Der Operator vor allem fungiert hier als eine Art sprachliches Achtung-Schild, das Sprecherin 4067 im übertragenen Sinne hochhält, um die Aufmerksamkeit ihres Gegenübers zu erhöhen und zu signalisieren, dass nun ein entscheidender Einwand folgt.

Die exemplarisch angeführten Gesprächsausschnitte aus Freundesgesprächen unseres Korpus spiegeln wider, wie SprecherInnen mittels Operatoren dem kommunikativen Status ihrer ÄuBerung Ausdruck verleihen. Die pragmatische Funktion des Operators tritt in den diskutierten Beispielen in Form von Versicherungen, Relativierungen, Unterstreichungen der Relevanz, der Sicherheit, des Wahrheitsgehalts oder der Offenheit der Äußerungen im Skopus in Erscheinung. GesprächspartnerInnen wollen sich richtig verstanden wissen und verfolgen daher die Intention, dass ihre Aussagen richtig eingeschätzt beziehungsweise interpretiert werden. $\mathrm{Zu}$ diesem Zweck können sie Operatoren mit projektiver Kraft einsetzen, die den kommunikativen Status der nachfolgenden Äußerung im Skopus charakterisieren.

\subsubsection{Operatoren zur Charakterisierung des mentalen Status}

Zur Charakterisierung des mentalen Status einer Äußerung im Skopus dienen Operatoren wie meine Meinung/Überzeugung, ich finde, ich meine, ich glaube, ich weiß etc. Nomen, nominaler Komplex oder Matrixsatz mit asyndetischem Anschluss der Äußerung mit Verbzweitstellung können in Operatorfunktion den mentalen Status verdeutlichen. Sie geben eine Verstehensanleitung im Hinblick darauf, welche psychisch-mentale Qualität die Äußerung im Skopus einnimmt (cf. Fiehler et al. 2004: 263). Dies kann eine Meinung, eine (Ein-)Schätzung, Wissen, eine Präferenz oder Ähnliches sein. Ohne Operator ist es dem Gegenüber überlassen, die Äußerung entsprechend zu interpretieren, da die Aussage an sich keine Schlüsse über den mentalen Status zulässt.

Aus unserem Korpus werden im Folgenden kurze Gesprächsausschnitte zur Verdeutlichung angeführt und näher diskutiert. Gerade unter den Operatoren zur Charakterisierung des mentalen Status gibt es zahlreiche Belege in den von uns analysierten Daten zu verzeichnen. Besonders häufig kommt allerdings (ich) meine in unterschiedlicher Realisierung vor (als ich meine, ich mein, $i$ meine, i mein, i maan, maan). Daher soll als erstes aus diesem Bereich ein Beispiel aufgegriffen werden.

\section{Beispiel (1) ich meine}

Imo (cf. 2007: 9) hält speziell zu ich mein(e) fest, dass es sich dann um einen Diskursmarker handelt, wenn die Phrase weglassbar, im Vorvorfeld positioniert und semantisch verblasst ist sowie eine pragmatische Funktion erfüllt. Das deckt sich auch mit den von uns aufgestellten Kriterien für Operator-Skopus-Strukturen: Der Operator allein ist keine selbstständige interaktive Einheit, ist prosodisch oder syntaktisch abgehoben und hat projektive Kraft auf die nachfolgende Äußerung. Dazu muss er nach unserer Definition eine Leerstelle eröffnen und in Kombination mit dem Skopus eine zweigliedrige, asyndetische Struktur bilden sowie eine interaktive Einheit konstituieren. 
Zunächst folgt eine Übersicht der alters- und geschlechtsmäßigen Verteilung des Operators ich meine (siehe Tabelle 7).

\begin{tabular}{|l|r|r|r|}
\hline \multicolumn{5}{|c|}{ ich meine } \\
\hline männlich & alt & jung & Gesamtanzahl \\
\hline weiblich & 293 & 89 & $n=386$ \\
\hline total & 158 & 132 & $n=286$ \\
\hline
\end{tabular}

Tabelle 7: Verteilung von ich meine nach Alter und Geschlecht

Operator-Skopus-Strukturen mit ich meine sind in unserem Korpus in jeder Alters- und Geschlechtsgruppe sehr häufig anzutreffen.

Folgender Gesprächsausschnitt aus einem Interview mit einem jungen Sprecher aus Wien zeigt eine solche Verwendung von ich meine deutlich auf. Die Interviewerin fragt Sprecher 4036 nach Spezifika der in Wien gebräuchlichen dialektalen Sprachform. ${ }^{8}$ Sprecher 4036 ist etwas unsicher in den Antworten und verwendet den Operator ich mein als Relativierung seiner Aussagen.

[1]

\begin{tabular}{lll}
$1142[25: 05.0]$ & $1143[25: 08.7]$ \\
\hline Expl [v] & $(---)$ fallen ihnen SONST irgendwelche bezeichnungen für das ein, $=$ wie in [wien]O
\end{tabular}

[2]

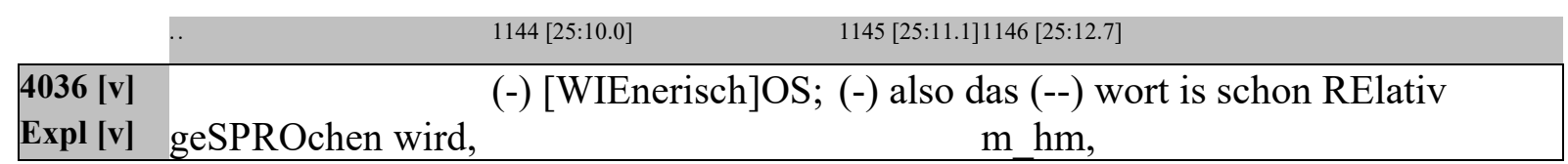

[3]

\begin{tabular}{|llll|}
\multicolumn{1}{l}{$1147[25: 13.0]$} & $1148[25: 14.2]$ & $1149[25: 18.3]$ \\
\hline 4036 [v] & gebräuchlich, & (4.8) also \\
Expl [v] & $(1.1)$ und was macht dieses [wienerisch]OS (-) AUS, & \\
\hline
\end{tabular}

[4]

$1150[25: 24.1]$ $1152[25: 25.7]$

4036 [v] [W:IEnerisch]OS, =und [wiener diaLEKT]OS- =hätt ich jetzt mal geSAGT, =is (.)

[5]

\begin{tabular}{|c|c|c|c|}
\hline & $1153[25: 26.6]$ & $1154[25: 27.1]$ & $1155[25: 29.1]$ \\
\hline $\begin{array}{l}4036[v] \\
\text { Expl [v] }\end{array}$ & dasSELbe, & & $=$ ich kenn (-) WEnig \\
\hline
\end{tabular}

[6]

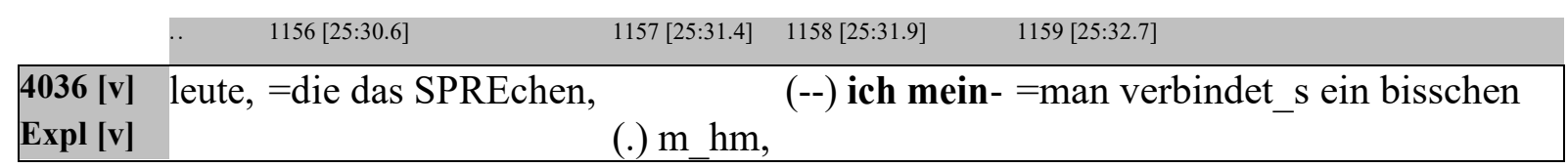

\footnotetext{
${ }^{8}$ Die Interviewerin ist in den Transkripten immer mit dem Sprecherkürzel Expl (als Abkürzung für Exploratorin) gekennzeichnet.
} 


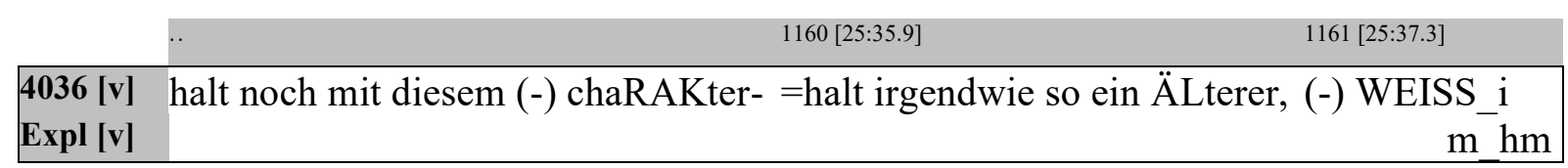

$[8]$

\begin{tabular}{|l|l|} 
& .. 1163 [25:38.2]1164 [25:38.4] \\
\hline $\mathbf{4 0 3 6}[\mathbf{v}]$ & nicht, $\quad(-)$ SATtelfester, \\
Expl [v] - &
\end{tabular}

(ULA-Korpus, 4036_WIER_jung_m_AI, Seg. 1142-1161)

Sprecher 4036 verweist auf seine seiner Einschätzung nach unzureichende Expertise, um eine fundierte Aussage über „Wienerisch“ treffen zu können. Er begründet das damit, wenige Menschen zu kennen, die diese Sprechweise verwenden. Um dieses Eingeständnis zu unterstützen, verwendet er ich mein. Mittels dieser Phrase will der Sprecher betont wissen, dass er dabei eine vorsichtige Prognose - eben im Sinne einer Meinung, nicht Wissen - formuliert. Er nimmt sich damit bewusst zurück, was durch das anschließende ,ich muss ehrlich sagen“ noch verstärkt wird. Gleich in der nächsten Äußerung kommt nochmals ich mein als Operator zum Einsatz, um eine allgemeinere Einschätzung bzw. Meinung mit dem Indefinitpronomen man abzugeben. Der Sprecher verweist auf gewisse Stereotype, die die Sprechweise an einen bestimmten Charaktertypus knüpfen - in diesem Fall ordnet er Wienerisch einem eher älteren, bodenständigen Sprecher zu. Der Operator nimmt also eine relativierende, einräumende Funktion ein und soll so dem Gegenüber signalisieren, dass die getätigte Aussage einer individuellen Einschätzung entspricht und keiner allgemeingültigen Feststellung oder Definition von Wienerisch oder SprecherInnen dieser Sprechweise. Formal auffällig ist, dass in beiden Vorkommen der Operator jeweils mit einer Pause (Dauer zwischen 0.5 und 0.75s) prosodisch abgesetzt wird.

Die Häufigkeit der Operator-Verwendung durch Sprecher 4036 mag an der Gesprächssituation beziehungsweise an der Personenkonstellation liegen. Da sich Sprecher und Interviewerin nicht kennen und dadurch Meinungen, Überzeugungen oder Wissen nicht bekannt sind, könnte Sprecher 4036 besonderes Interesse an der Rahmung seiner Äußerungen haben. So kann er sicherstellen, dass seine Aussagen von der Interviewerin richtig eingeordnet werden. Indem er ihr eine Interpretationshilfe zur Verfügung stellt, wird Missverständnissen vorgebeugt. Außerdem könnte eine Rolle spielen, dass Sprecher 4036 die Interviewerin als Expertin wahrnimmt, vor der er nichts Falsches oder Unzutreffendes im Interview sagen möchte. Auch dazu dient der Operator ich mein, indem er die Sicherheit beziehungsweise Bestimmtheit der Aussage abschwächt. Sprecher 4036 verwendet diese Strategie weitere Male über das Interview hinweggenau genommen gibt es 22 Vorkommen dieses Operators in seinen Sprachdaten.

\section{Beispiel (2) ich glaube}

Im nächsten Beispiel soll der am zweithäufigsten auftretende Operator zur Charakterisierung des mentalen Status aufgegriffen werden. Dabei handelt es sich um die kurze Phrase ich glaube, die als Verstehenshinweis für die nachfolgende Äußerung im Skopus eingesetzt wird.

Tabelle 8 führt an, wie die SprecherInnengruppen verteilt nach Alter und Geschlecht ich glaube in Operatorfunktion verwenden: 


\begin{tabular}{|l|r|r|r|}
\hline \multicolumn{4}{|c|}{ ich glaube } \\
\hline & alt & jung & Gesamtanzahl \\
\hline männlich & 55 & 86 & $n=141$ \\
\hline weiblich & 65 & 75 & $n=140$ \\
\hline total & 120 & 161 & $n=281$ \\
\hline
\end{tabular}

Tabelle 8: Verteilung von ich glaube nach Alter und Geschlecht

Die tabellarische Übersicht zeigt, dass die SprecherInnengruppen ähnliche Verwendungshäufigkeiten aufweisen.

Folgender Transkriptausschnitt stammt aus einem Interview mit einer jungen Grazer Sprecherin. Die Interviewerin möchte wissen, was Sprecherin 4070 tun würde, wäre sie Bürgermeisterin der Stadt Graz. Obwohl die Sprecherin einräumt, kein politisches Amt ausüben zu wollen, hat sie doch Ideen, was sie gegebenenfalls umsetzen würde.

[1]

$$
550 \text { [17:01.3] }
$$

$\operatorname{Expl~[v]~(-)~ahm~(--)~angenommen~sie~könnten~von~heute~auf~morgen~plötzlich~}$

[2]

\begin{tabular}{|lll|}
\hline $\begin{array}{l}\text { Expl [v] } \\
\text { 4070 [v] }\end{array}$ & BÜRgermeisterin (.) von [graz]O sein; $\quad$ gäbe es da IRgendetwas (--) das sie ( 552 [17:07.9] \\
\hline
\end{tabular}

[3]

\begin{tabular}{|c|c|c|}
\hline & $553[17: 11.8]$ & 554 [17:13.3] \\
\hline $\begin{array}{l}\operatorname{Expl}[v] \\
4070[v]\end{array}$ & -) verändern würden, (-) oder das sie ANpacken würden, & (2.2) ja aiso i i bin ehrlich \\
\hline
\end{tabular}

[4]

4070 [v] gsagt sehr froh dass i ka poLItiker bin, $=$ wei:1 ich glaub (.) das stellt ma si vielleicht [5]

$$
556 \text { [17:22.0] } 557 \text { [17:23.9] }
$$

4070 [v] a bissi EINfacher vor, als es dann in der realiTÄT is, (--) weil ma muss es ja am

[6]

4070 [v] schluss doch allen RECHT machen; (--) aber ANgenommen ich wär jetzt

[7]

\begin{tabular}{|cc|}
\multicolumn{1}{c}{$559[17: 28.8]$} & $560[17: 32.4] 561[17: 32.6]$ \\
\hline $\begin{array}{l}\text { Expl [v] } \\
\mathbf{4 0 7 0}[\mathbf{v}]\end{array}$ & $<<$ bichernd $>$ ja- $>$ \\
\hline
\end{tabular}

\begin{tabular}{|c|c|c|}
\hline & $562[17: 33.0] 563[17: 35.2]$ & 564 [17:36.3] \\
\hline Expl [v] & & \\
\hline $4070[v]$ & kane NEgativen konsequenzen geben, (-) dann & (1.0) an riesen riesen \\
\hline
\end{tabular}

[8] 
[9]

\begin{tabular}{|ll} 
Expl [v] \\
$\mathbf{4 0 7 0}[\mathbf{v}] \quad$ sack voll GELD $<<$ kichernd $>$ der universität vor die tür stellen, $>$
\end{tabular}$\quad$\begin{tabular}{l}
$565[17: 40.7]$ \\
\hline
\end{tabular}

$[10]$

\begin{tabular}{|c|c|} 
& $566[17: 41.2] 567[17: 41.8]$ \\
\hline $\begin{array}{l}\text { Expl [v] } \\
\text { 4070 [v] }\end{array}$ & SEHR schön, =das kann ich nur beGRÜßen,> \\
\hline
\end{tabular}

(ULA-Korpus, 4070_GRAG_jung_w_AI, Seg. 550-567)

Die Sprecherin gibt in ihrer Äußerung zu bedenken, dass die Ausübung eines politischen Amtes in der Praxis wohl schwieriger sei, als man es sich gemeinhin vorstellen würde. Dieser Zwischenkommentar, der sich über die Operator-Skopus-Struktur erstreckt, erfüllt den Zweck der Relativierung der nachfolgenden Ausführungen, womöglich um der Interviewerin nicht zu nahe zu treten. Sprecherin 4070 formuliert ihre Aussage zurückhaltend und vorsichtig, beginnend mit dem relativierenden Operator ich glaube und anschließend mit der adverbialen Verwendung von vielleicht im Skopus. Dies unterstreicht die Tatsache, dass die Sprecherin keine allgemeingültige Aussage tätigen möchte, sondern vielmehr dem Gegenüber signalisieren will, dass es sich um eine persönliche Einschätzung handelt. Die Interviewerin bekommt damit Hilfestellung, wie die Aussage zu interpretieren ist. Die Mikropause zwischen Operator und Skopus setzt ersteren prosodisch von letzterem ab und unterstreicht so formal die zweigliedrige Struktur.

\section{Beispiel (3) ich finde}

Als drittprominenteste Gruppe der Operatoren zur Charakterisierung des mentalen Status tritt in unseren Sprachdaten ich finde in Erscheinung. Wie sich die 153 Belege auf die SprecherInnengruppen verteilen, wird in Tabelle 9 deutlich:

\begin{tabular}{|l|r|r|r|}
\hline \multicolumn{5}{|c|}{ ich finde } \\
\hline & alt & jung & Gesamtanzahl \\
\hline männlich & 11 & 45 & $n=57$ \\
\hline weiblich & 16 & 81 & $n=96$ \\
\hline total & 27 & 126 & $n=153$ \\
\hline
\end{tabular}

Tabelle 9: Verteilung von ich finde nach Alter und Geschlecht

Folgender Gesprächsausschnitt stammt aus einem Interview mit einem älteren Sprecher aus der Grazer Umlandgemeinde Kalsdorf. Es geht thematisch um die Frage, wie LehrerInnen im Unterricht mit SchülerInnen sprechen sollten beziehungsweise welche Sprechweise angemessen wäre. Sprecher 4032 vertritt die Auffassung, dass LehrerInnen in ihrer Vorbildfunktion Wert auf ,gutes“, korrektes Standarddeutsch legen sollten.

[1]

1211 [31:01.1]

Expl [v] (1.5) und ahm wie sollten ihrer meinung nach LEHrer beziehungsweise lehrerinnen 
[2]

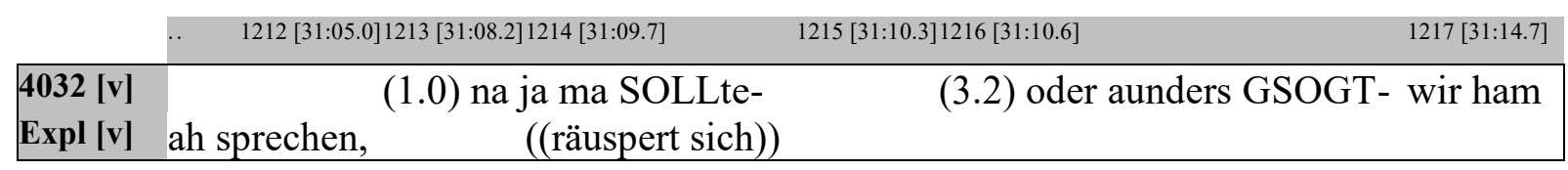

[3]

1218 [31:15.2]1219 [31:18.0]

4032 [v] (--) auch in den höheren schulen DERzeit- (1.1) sicher an die DREIßich $m$ bis

[4]

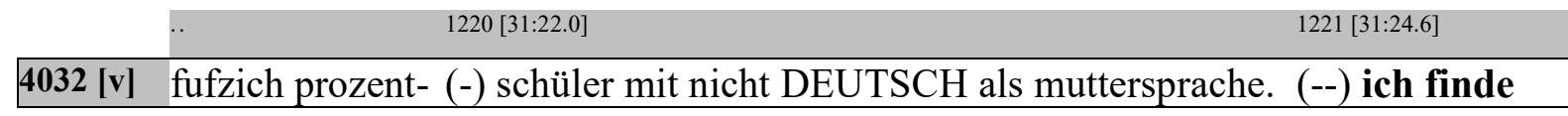

[5]

4032 [v] schon aus der BEIspüwirkung heraus sollten die lehrer- (--) HOIwegs gut deutsch [6]

\begin{tabular}{|c|c|c|}
\hline & $1223[31: 30.8]$ & $1224[31: 33.0]$ \\
\hline $4032[v]$ & war grammatikalisch in ganzen SÄTzen, & BROckenwe \\
\hline Expl [v] & & $\mathrm{m}$ \\
\hline
\end{tabular}

[7]

\begin{tabular}{|l|l|}
$1226[31: 34.1]$ & $1227[31: 34.4] 1228[31: 37.3]$ \\
\hline $\begin{array}{l}\text { 4032 [v] } \\
\text { Expl [v] }\end{array}$ & $(--)$ wal ma kann etwas nur weitergeben wenn ma_s aa VORlebt; (--) und des \\
\hline
\end{tabular}

[8]

1229 [31:38.6] 1230 [31:40.4]

4032 [v] SOLLte man; =i glaub d d äh es gibt heit NIEmanden- =der net durch des beispiel

[9]

4032 [v] am LEIchtesten und am schnösten lernt; (wos) WURSCHT wos er vorher können [10]

4032 [v] hot;

(ULA-Korpus, 4032_KALSD_alt_m_AI, Seg. 1211-1231)

Sprecher 4032 plädiert für die Verwendung von ,gutem“ Deutsch im Unterricht. Dies soll seiner Meinung nach einerseits dazu dienen, dass SchülerInnen mit einer anderen L1 dem Unterricht besser folgen können und andererseits sollen sich alle SchülerInnen ein Beispiel an der Lehrperson nehmen können und folglich einen besseren Lernerfolg erzielen. Um deutlich zu machen, dass es sich dabei um seine persönliche Einschätzung handelt, verwendet Sprecher 4032 äußerungsinitial den Operator ich finde. Er drückt damit aus, dass er seine Überzeugung im Skopus der Äußerung wiedergibt, während er ihr gleichermaßen Ausdruck verleiht. Die Interviewerin weiß also seine Äußerung entsprechend einzuordnen und zu interpretieren. Da sich die Gesprächspartner des Interviews nicht kennen, ist eine solche Verstehensanleitung für den Sprecher ein adäquates Mittel, um seinem Gegenüber den Stellenwert seiner Aussage näher zu bringen. 


\section{Beispiel (4) ich weiß}

Die vierte Phrase mit Operatorfunktion, die häufig in unserem Korpus zu finden ist, ist ich weiß. Das Vorkommen des Operators ich weiß lässt sich sortiert nach den sozialen Faktoren Alter und Geschlecht in unserem Korpus in folgenden Frequenzen (siehe Tabelle 10) erfassen:

\begin{tabular}{|l|r|r|r|}
\hline \multicolumn{5}{|c|}{ ich wei $\boldsymbol{\beta}$} \\
\hline & alt & jung & Gesamtanzahl \\
\hline männlich & 8 & 6 & $n=14$ \\
\hline weiblich & 25 & 19 & $n=44$ \\
\hline total & 33 & 25 & $n=58$ \\
\hline
\end{tabular}

Tabelle 10: Verteilung von ich weiß nach Alter und Geschlecht

Nachfolgender Gesprächsausschnitt zeigt den Einsatz von ich weiß als Operator durch eine ältere Sprecherin aus Wien. Im vorliegenden Interview thematisiert die Sprecherin ihre altersbedingten körperlichen Einschränkungen, die ihr auch die Ausübung ihres liebsten Hobbys - der Malerei - erschweren.

$[1]$

\begin{tabular}{|c|c|c|}
\hline & 615 [18:47.5] & $616[18: 48.3]$ \\
\hline $4039[\mathrm{v}]$ & (-) das fehlt mir jetzt toTAL, weil das GEHT net; & ma kann net mit malrucksock und \\
\hline
\end{tabular}

\begin{tabular}{|c|c|c|}
\hline & $617[18: 50.2] 618$ [18:50.7] & 619 [18:53.9] \\
\hline Expl [v] & $\mathrm{m} \mathrm{hm-}$ & \\
\hline
\end{tabular}

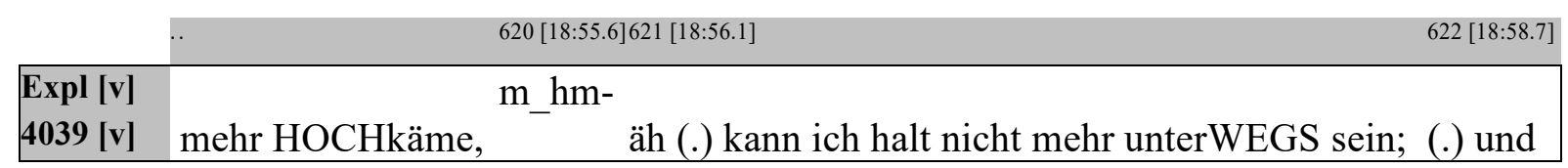

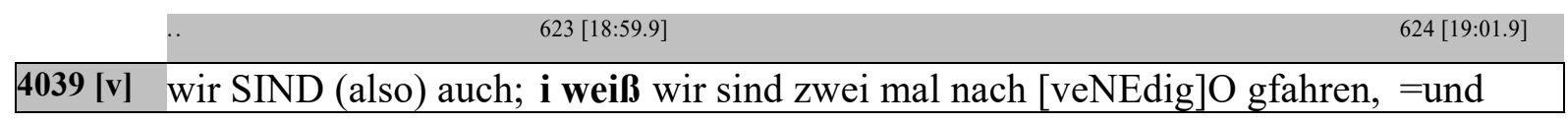
$[5]$

\begin{tabular}{|lcc|}
\hline Expl [v] & $625[19: 03.8]$ & $626[19: 04.7] 627[19: 05.9]$ \\
\hline $\mathbf{4 0 3 9}$ [v] ham uns dort eine WOHnung gemietet, & $(-)$ zu FÜNFT, (-) und wirklich (-) ä:h zu \\
\hline
\end{tabular}

[6]

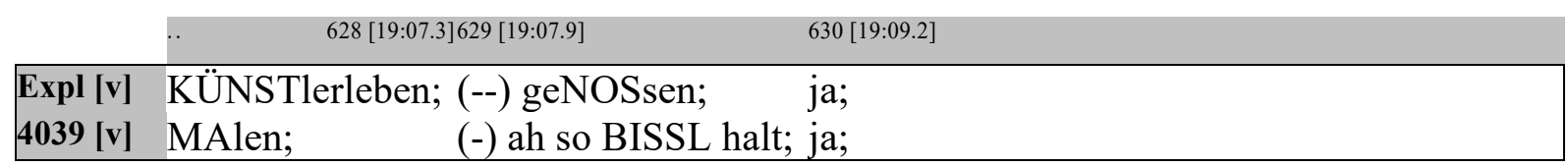

(ULA-Korpus, 4039_WIER_alt_w_AI, Seg. 614-630)

In diesem Beispiel schwelgt Sprecherin 4039 in Erinnerungen an jene Zeit, in der sie ihre Hobbys noch uneingeschränkt ausüben konnte. Einleitend weist sie darauf hin, dass es ihr nicht mehr möglich ist, der Malerei nach ihren Wünschen und Vorstellungen nachzugehen, da es ihr 
gesundheitlicher Zustand nicht zulässt. Mit $i$ weiß in äußerungsinitialer Position leitet sie zu früheren Erlebnissen über, als sie noch reisen und malen konnte. Der Operator wird in diesem Sinne als verbindendes Glied zwischen Gegenwart und Zukunft eingesetzt. Er drückt somit einerseits aus, dass sich die Sprecherin noch gut und gerne an frühere Zeiten erinnert, und bekräftigt andererseits den Wahrheitsgehalt ihrer Aussage. Es soll bei der Interviewerin keinerlei Zweifel an der Verlässlichkeit ihres Erinnerungsvermögens entstehen. Da sich die Gesprächspartner im Interviewsetting nicht kennen, stellt die Phrase einen nützlichen Interpretationshinweis mit projektiver Kraft dar. Er hilft der Interviewerin, die Äußerung von Sprecherin 4039 richtig einzuordnen und so ein besseres Bild von ihr zu bekommen.

Alle angeführten Beispiele von Operatoren zur Charakterisierung des mentalen Status von Äußerungen, die sich in den Gesprächsausschnitten der Interviews unseres Korpus finden, zeigen deutlich, dass die SprecherInnen diese gezielt zur Verständnissicherung einsetzen. Die Operatoren fungieren als Interpretationshilfe, die es der dem Sprecher/der Sprecherin unbekannten Interviewerin ermöglicht, die Äußerungen richtig einzuordnen. Die SprecherInnen nutzen Operator-Skopus-Strukturen beispielsweise dazu, ihre Aussagen zu relativieren, den Wahrheitsgehalt oder die Verlässlichkeit zu bekräftigen, Einschränkungen auszudrücken oder aber anzuzeigen, dass es sich um ein Eingeständnis, eine persönliche Einschätzung oder feste Überzeugung handelt. Im Setting des Interviews ist es natürlich für die Interviewten von besonderem Interesse, über sich Auskunft zu geben und dabei richtig verstanden zu werden.

\section{Diskussion der Ergebnisse}

Die angeführten Beispiele im vorhergehenden Kapitel zeigen, wie SprecherInnen gezielt Operator-Skopus-Strukturen verwenden, um den mentalen oder kommunikativen Status einer Äußerung zu charakterisieren. Auch wenn die Gesprächsausschnitte, welche Operatoren beinhalten, die zur Charakterisierung des kommunikativen Status dienen, alle aus Freundesgesprächen stammen, bedeutet das nicht, dass diese auf das informellere Setting beschränkt sind. Im Gegenteil - sie finden sich ebenso in den Interviews und erfüllen dort ähnliche Funktionen in der Interaktion. Gleiches gilt für die Operatoren zur Charakterisierung des mentalen Status. Sie kommen in beiden Settings zum Einsatz, wenn auch die ausgewählten Beispiele die Verwendung in der Interviewsituation abbilden.

Das Setting (Interview oder Freundesgespräch) der Interaktion, das den sozialen Raum des Gesprächs definiert, kann jedoch sehr wohl Einfluss auf die Wahl der Operatoren und die Frequenz ihrer Verwendung nehmen. Abhängig beispielsweise von der Art der (sozialen) Beziehung zwischen den beiden GesprächspartnerInnen, vom Kontext der Äußerung, vom gemeinsamen/geteilten (Welt-)Wissen, von der Alters- oder Geschlechtsdifferenz können SprecherInnen über die Notwendigkeit eines Verstehenshinweises in Form eines Operators entscheiden. Auch die Hierarchie der GesprächspartnerInnen kann ausschlaggebend sein. Gerade im Interviewsetting, in welchem die Interviewerin die Rolle der Expertin einnimmt, mag die interviewte Person ein besonderes Interesse daran haben, ihre Äußerungen entsprechend zu rahmen, um sich verstanden zu wissen - einerseits, um einen guten Eindruck beim Gegenüber zu hinterlassen und andererseits, um Missverständnissen vorzubeugen. Da sich beide SprecherInnen im formell konzipierten Interview unbekannt sind und siezen, gilt es hier, über das Gespräch eine gemeinsame 
Ebene zu finden. Sprachliche Mittel, die zu gegenseitigem Verständnis und besserer Interpretierbarkeit des Gesagten führen, sind dafür ein wichtiges und nützliches Instrument.

Doch auch im Freundesgespräch ist diese pragmatische Funktion von Bedeutung. Zwar steht hier vielleicht weniger im Fokus, dass sich die GesprächspartnerInnen besser kennen und einschätzen lernen (obwohl dieser Faktor wohl immer eine Rolle spielt), doch auch in dieser Konstellation ist es den SprecherInnen wichtig, ihre kommunikative Absicht klar zu äußern und somit richtig verstanden zu werden. Sei es, dass vom Gegenüber eine konkrete Reaktion oder Handlung erwartet wird, oder um eine Aussage abzuschwächen, um das Gesicht des anderen zu wahren. Es kann aber auch die Sicherheit beziehungsweise Verlässlichkeit der Äußerung unterstrichen, der Wahrheitsgehalt versichert oder einer Aussage ein spezieller Status zugewiesen werden (z. B. der einer Überzeugung, einer Einschätzung/Meinung, Einräumung etc.).

Zum Einfluss des Settings auf den Gebrauch von Operator-Skopus-Strukturen in unseren Sprachdaten können wir keine genaueren Aussagen treffen, da das Korpus von 93 Gesprächen lediglich 20 Gespräche aus einem informellen Setting enthält und die Vorkommen der Operatoren zum Teil so gering sind, dass keine Vergleiche der Ergebnisse aus dem formellen versus dem informellen Setting angestellt werden können. Auch muss darauf hingewiesen werden, dass sich bestimmte Trefferzahlen auf einzelne Individuen und nicht verteilt auf mehrere oder gar alle SprecherInnen zurückführen lassen. Dies unterstützt die Annahme, dass es sich dabei eher um individuelle Verwendungstendenzen handelt als um gruppenspezifische Präferenzen.

So unterschiedlich die Gebrauchsfrequenzen und Präferenzen unter den SprecherInnen auch sein mögen, zeigt sich doch eine Übereinstimmung in der intendierten pragmatischen Funktion der Operatoren. Die angeführten Gesprächsausschnitte bilden zwar jeweils nur ein exemplarisches Vorkommen der beschriebenen Operator-Skopus-Struktur ab, jedoch stehen sie stellvertretend für alle gefundenen Operator-Belege. Natürlich werden die Operatoren von den SprecherInnen nicht in jedem Gespräch mit genau derselben Intention eingesetzt, sondern erfahren bestimmte Modifizierungen, die beispielsweise angepasst an das Gesprächsthema, die Gesprächssituation oder den Gesprächspartner/die Gesprächspartnerin erfolgen. Dennoch bleiben die grundlegenden pragmatischen Funktionen des Operators - nämlich die der Diskursorganisation und Bereitstellung einer Verstehensanweisung für das Gegenüber - in allen Vorkommen von Operator-Skopus-Strukturen in unserem Korpus klar erkennbar.

Wie von Imo (cf. 2007) zu ich mein(e) beschrieben, verblasst die Semantik der Diskursmarker in vielen Fällen. Das zeigt im Fall von ich mein(e) die Umformungsprobe in einen Matrixsatz mit eingeleitetem Nebensatz. Eine solche Umformung würde nämlich eine semantische Veränderung zur Folge haben beziehungsweise die semantische Bedeutung (z. B. von meinen) wieder aktivieren (cf. Imo 2007: 8). Bezogen auf unsere Analyse sind dahingehend besonders die Operator-Skopus-Strukturen mit ich meine, ich finde und ich weiß zu erwähnen. Diese Phrasen stellen nicht nur die zahlenmäßig größte Gruppe unter den Operatoren dar, sondern zeigen sich auch fast ausschließlich in Operatorfunktion. Dies lässt sich wohl auf den Umstand zurückführen, dass sie bereits vielfach semantisch verblasst und daher auf die pragmatische Funktion reduziert bzw. konzentriert sind. 


\section{$5 \quad$ Schluss}

Zusammenfassend kann festgehalten werden, dass SprecherInnen unseres Korpus OperatorSkopus-Strukturen zu unterschiedlichen Zwecken, aber immer mit pragmatischer Funktion im Diskurs verwenden. Sie nutzen sie in äußerungsinitialer oder insertierter Position mit oder ohne zusätzliche prosodische Markierung im formellen Setting des Interviews ebenso wie im informellen Setting des Freundesgesprächs. Es wird deutlich, dass der Einsatz von Operatoren zur Charakterisierung des kommunikativen Status wie ehrlich, wirklich, sicher(lich), vor allem insbesondere darauf abzielt, dem Gesprächspartner/der Gesprächspartnerin den Wahrheitsgehalt der Äußerung zu vermitteln, die Sicherheit, Verlässlichkeit oder Belastbarkeit einer Aussage hervorzuheben, ihre Bedeutung zu unterstreichen, aber auch eine Einschränkung oder Einräumung sowie eine Relativierung vorzunehmen. Die Operatoren zur Charakterisierung des mentalen Status wie ich mein, ich glaube, ich finde oder ich weiß dagegen signalisieren vorwiegend, ob es sich beispielsweise um eine Meinung, Einschätzung, Überzeugung oder ein Eingeständnis handelt. Sie bieten eine Interpretationshilfe, um den Gehalt der Äußerung richtig einordnen zu können und so das Verständnis zu sichern. Die hier diskutierten Operatoren übernehmen dabei oftmals nicht nur eine einzige pragmatische Funktion im Gespräch. SprecherInnen vermitteln durch sie mehrere Aspekte ihrer Sprecherintention und reichern damit den Äußerungsgehalt ihrer Aussage an, um dem Gegenüber eine hinreichende Verstehensanleitung der Äußerung im Skopus zur Verfügung zu stellen. Die Wahl der konkreten Operatoren hängt dabei stark von der Gesprächssituation sowie der Art der Beziehung der GesprächspartnerInnen (Vertrautheit, gemeinsames Wissen, Hierarchie etc.) ab.

Da unser Korpus sehr heterogen ist, i. e. die Verteilung der SprecherInnen nach Alter und Geschlecht nicht ausgewogen, können keine Vergleiche der Verwendungsfrequenzen oder -präferenzen angestellt werden. Fraglich bleibt allerdings, ob ein solcher Vergleich mit einem homogenen Korpus anzustreben wäre, da es sich streckenweise um ganz individuelle Präferenzen und Verwendungsfrequenzen von Operator-Skopus-Strukturen handelt, sodass ein interindividueller Vergleich in unseren Augen ohnehin nicht zielführend wäre. Entsprechend scheint es lohnender, weiter auf intraindividuelle Verwendungsweisen zu fokussieren. Dazu empfiehlt sich für weitere Untersuchungen, eine interaktionale Tiefenanalyse vollständiger Gespräche einzelner SprecherInnen vorzunehmen. Es ließe sich dadurch prüfen, ob noch andere und, wenn ja, welche Lexeme beziehungsweise Phrasen als Operatoren in den Gesprächen eingesetzt werden, um den mentalen oder kommunikativen Status einer Äußerung im Skopus zu charakterisieren.

\section{Literaturverzeichnis}

Altmann, Hans (1981): Formen der „Herausstellung “im Deutschen. Tübingen: Niemeyer.

Auer, Peter (1997): „Formen und Funktionen der Vor-Vorfeldbesetzung im gesprochenen Deutsch“. In: Schlobinski, Peter (ed.): Syntax des gesprochenen Deutsch. Opladen, Westdeutscher Verlag GmbH: 55-91.

Barden, Birgit/Elstermann, Mechthild/Fiehler, Reinhard (2001): „Operator-Skopus-Strukturen in gesprochener Sprache“. In: Liedtke, Frank/Hundsnurscher, Franz (eds.) Pragmatische Syntax. Tübingen, Niemeyer: 197-233. (= Beiträge zur Dialogforschung 23). 
Brinton, Laurel J. (1988): „'The flowers are lovely; only they have no scent'. The Evolution of a Pragmatic Marker". In: Borgmeier, Raimund/Grabes, Hubert/Jucker, Andreas H. (eds.): Proceedings of the Conference of the German Association of University Teachers of English. Vol XIV. Trier/Giessen, Wissenschaftlicher Verlag: 9-33.

Couper-Kuhlen, Elizabeth/Selting, Margret (2001). „Introducing interactional linguistics”. In: Selting, Margret/Couper-Kuhlen, Elizabeth (eds.): Studies in interactional linguistics. Amsterdam, Benjamins: 1-22.

Croft, William (2001): Radical Construction Grammar. Syntactic Theory in Typological Perspective. Oxford: University Press.

Deppermann, Arnulf (2006): „Construction Grammar - eine Grammatik für die Interaktion?“. In: Deppermann, Arnulf/Fiehler, Reinhard/Spranz-Fogasy, Thomas (eds.): Grammatik und Interaktion. Radolfzell, Verlag für Gesprächsforschung: 43-65.

Erman, Britt (1987): Pragmatic Expressions in English. Stockholm: Almqvist \& Wiksell International.

Fiehler, Reinhard (2005): „Gesprochene Sprache“. In: Duden. Die Grammatik. 7. Auflage. Mannheim etc.: Dudenverlag: 1175-1256.

Fiehler, Reinhard et al. (2004): Eigenschaften gesprochener Sprache. Tübingen: Narr. (= Studien zur Deutschen Sprache 30).

Fischer, Kerstin (2014): „Discourse Markers“. In: Barron, Ann/Schneider, Klaus (eds.): Pragmatics of Discourse. Handbooks of Pragmatics Vol. 3. Verlin/NewYork, de Gruyter: 271-294.

Fraser, Bruce (1990): „An Approach to Discourse Markers”. Journal of Pragmatics 14: 383-395.

FWF, F 60 (Fonds zur Förderung der wissenschaftlichen Forschung): „Deutsch in Österreich (DiÖ). Variation - Kontakt - Perzeption“. fwf.ac.at/de/wissenschaft-konkret/im-fokusschwerpunkte/f60-alexandralenz [08.10.2021].

Gohl, Christine/Günthner, Susanne (1999): „Grammatikalisierung von weil als Diskursmarker in der gesprochenen Sprache“. Zeitschrift für Sprachwissenschaft 18: 39-75.

Gülich, Elisabeth (1970): Makrosyntax der Gliederungssignale im gesprochenen Französisch. München: Fink (Structura).

Günthner, Susanne/Imo, Wolfgang (2003): „Die Reanalyse von Matrixsätzen als Diskursmarker: ich mein-Konstruktionen im gesprochenen Deutsch“. Interaction and Linguistic Structures 37.

Helbig, Gerhard/Buscha, Joachim (2001): Deutsche Grammatik. Ein Handbuch für den Ausländerunterricht. Berlin/New York: Langenscheidt.

Hennig, Mathilde (2006): Grammatik der gesprochenen Sprache in Theorie und Praxis. Kassel: Kassel University Press GmbH.

Imo, Wolfgang (2016): „Diskursmarker: grammatischer Status - Funktionen in monologischen und dialogischen Kontexten - historische Kontinuität“. Arbeitspapierreihe Sprache und Interaktion 65 (06/2016). arbeitspapiere.sprache-interaktion.de/65-wolfgang-imo-062016/ [07.10.2021].

Imo, Wolfgang (2012): „Wortart Diskursmarker?“. In: Rothstein, Björn (ed.): Nicht-flektierende Wortarten. Berlin etc., de Gruyter: 48-88. (=Linguistik - Impulse \& Tendenzen 47). 
Imo, Wolfgang (2007): „Zur Anwendung der Construction Grammar auf die gesprochene Sprache - der Fall ,ich mein(e)““. In: Ágel, Vilmos/Hennig, Mathilde (eds.): Zugänge zur Grammatik der gesprochenen Sprache. Tübingen, Niemeyer: 3-34. (= Reihe Germanistische Linguistik 269).

Jürgens, Frank (1999): Auf dem Weg zu einer pragmatischen Syntax. Eine vergleichende Fallstudie zu Präferenzen in gesprochen und geschrieben realisierten Textsorten. Tübingen: Niemeyer (= Reihe Germanistische Linguistik 207).

Kroon, Caroline (1995): Discourse Particles in Latin. Amsterdam: Gieben.

Lenk, Uta (1998): „Discourse markers and global coherence in conversation”. Journal of Pragmatics 30/2: 245-257.

Leska, Christel (1965): „Vergleichende Untersuchungen zur Syntax gesprochener und geschriebener deutscher Gegenwartssprache“. In: Beiträge zur Geschichte der deutschen Sprache und Literatur (Halle/Saale) 97, 427-464.

Pasch, Renate et al. (2003): Handbuch der deutschen Konnektoren. Berlin: de Gruyter.

Rupp, Heinz (1965): „Gesprochenes und geschriebenes Deutsch“. In: Wirkendes Wort 15: 19-29.

Schegloff, Emanuel A. (2007). Sequence organization in interaction: A primer in conversation analysis. Cambridge: Cambridge University Press.

Schegloff, Emanuel A./Ochs, Elinor/Thompson, Sandra A. (1996): „Introduction“. In: Ochs, Elinor/Schegloff, Emanuel A./Thompson, Sanda A. (eds.): Interaction and Grammar. Cambridge, CUP: $1-51$.

Schiffrin, Deborah (1987): Discourse Markers. Cambridge: University Press.

Schneider, Jan Georg/Butterworth, Judith/Hahn, Nadine (2018): Gesprochener Standard in syntaktischer Perspektive. Theoretische Grundlagen-Empirie-didaktische Konsequenzen. Tübingen: Stauffenburg. (= Stauffenburg Linguistik 99).

Schlobinski, Peter (1997): Syntax des gesprochenen Deutsch. Opladen: Westdeutscher Verlag.

Schröder, Peter (2006): „Das Vorforfeldkonzept aus gesprächsanalytischer Sicher - Plädoyer für eine handlungsorientierte Einheitenbildung in einer Grammatik der gesprochenen Sprache“. In: Deppermann, Arnulf/Fiehler, Reinhard/Spranz-Fogasy, Thomas (eds.). Grammatik und Interaktion. Radolfzell, Verlag für Gesprächsforschung: 203-243.

Schourup, Lawrence Clifford (1982): „Common Discourse Particles in English Conversation“. New York: Garland.

Schourup, Lawrence Clifford. (1999): „Discourse markers”. Lingua 10: 227-265.

Selting, Margret et al. (2009): „Gesprächsanalytisches Transkriptionssystem 2 (GAT 2)“. Gesprächsforschung - Online-Zeitschrift zur verbalen Interaktion 10: 353-402. gespraechsforschung-online.de/fileadmin/dateien/heft2009/px-gat2.pdf [30.11.2020].

Thim-Mabrey, Christiane (1988): „Satzadverbialia und andere Ausdrücke im Vorvorfeld“. Deutsche Sprache 16: 55-67.

Weinrich, Harald (1993): Textgrammatik der deutschen Sprache. Mannheim: Dudenverlag. 